\title{
An RNA dynamic ensemble at atomic resolution
}

Honglue Shi ${ }^{1}$, Atul Rangadurai ${ }^{2}$, Hala Abou Assi ${ }^{2,3}$, Rohit Roy ${ }^{4}$, David A. Case ${ }^{5 *}$, Daniel Herschlag $6,7,8 *$ Joseph D. Yesselman ${ }^{9 *}$, and Hashim M. Al-Hashimi ${ }^{1,2 *}$

${ }^{1}$ Department of Chemistry, Duke University, Durham NC 27710 USA

${ }^{2}$ Department of Biochemistry, Duke University School of Medicine, Durham NC 27710 USA

${ }^{3}$ Department of Medicine, Duke University School of Medicine, Durham NC 27710 USA

${ }^{4}$ Center for Genomics and Computational Biology, Duke University School of Medicine, Durham NC 27710 USA

${ }^{5}$ Department of Chemistry and Chemical Biology, Rutgers University, Piscataway NJ 08854 USA

${ }^{6}$ Department of Biochemistry, Stanford University, Stanford CA 94305 USA

${ }^{7}$ Department of Chemical Engineering, Stanford University, Stanford CA 94305 USA

${ }^{8}$ ChEM-H Institute, Stanford University, Stanford, CA 94305 USA

${ }^{9}$ Department of Chemistry, University of Nebraska-Lincoln, Lincoln, NE 68588 USA

*Correspondence to: hashim.al.hashimi@duke.edu, jyesselm@unl.edu, herschla@stanford.edu, david.case@rutgers.edu 


\section{Abstract:}

Biomolecules do not fold into a single 3D structure but rather form dynamic ensembles of many inter-converting conformations ${ }^{1}$. Knowledge of dynamic ensembles is key for understanding how biomolecules fold and function, and for rationally manipulating their activities in drug discovery and synthetic biology ${ }^{2-4}$. However, solving dynamic ensembles of biomolecules at atomic resolution is a major challenge in structural biology because the information required to specify the position of all atoms in thousands of conformations in an ensemble far exceeds the information content of experimental measurements. Here we addressed the data gap and dramatically simplified and accelerated RNA ensemble determination by using structure prediction tools that leverage the growing database of RNA structures to generate a conformational library. Library refinement with NMR residual dipolar couplings enabled determination of an atomicresolution ensemble for HIV-1 TAR as confirmed by quantum-mechanical calculations of NMR chemical shifts, comparison to a crystal structure of a substate, and through the successful redistribution of the ensemble by design using atomic mutagenesis. The ensemble provides an unprecedented view of how bulge residues cooperatively flip out and undergo sugar repuckering to allow the adjoining helices to stack. The generality of this approach will make determination of atomic-resolution RNA ensembles routine. 


\section{Main Text:}

The functions of many regulatory RNAs crucially depend on changes in threedimensional (3D) structure that occur in response to a diverse array of cellular inputs, including the binding of small-molecule ligands ${ }^{5,6}$, proteins ${ }^{7}$, epitranscriptomic modifications $^{8}$, mutations ${ }^{9}$, and even to nascent RNA elongation during transcription ${ }^{10,11}$. Initially described as changes from one 3D structure to another, these transitions are now better understood as changes in dynamic ensembles of many inter-converting conformations, from one conformational distribution to another ${ }^{4,12}$.

To deeply understand RNAs at a level that ultimately makes it possible to rationally manipulate their behavior in drug discovery and synthetic biology, we need an ability to determine their dynamic ensembles at atomic resolution. This however presents a significant challenge to current biophysical techniques. The information required to specify the position of all atoms in thousands of conformations in an ensemble far exceeds the information content of experimental measurements. NMR spectroscopy is a rich source of ensemble-averaged measurements, and in combination with computational modelling, has been applied with success to determine ensembles of proteins at atomic

resolution $^{13-15}$. In contrast, fewer atomic-level experimental measurements are typically available for characterizing RNA ensembles. Moreover, while computational modeling methods such as molecular dynamics (MD) simulations are needed to address the experimental data gap $^{16}$, nucleic acid force fields remain underdeveloped relative to proteins, and MD simulations of RNAs often poorly predict experimental data even for 
simple motifs ${ }^{17,18}$. Consequently, relative to proteins, there is a greater danger of overfitting an RNA ensemble, and assessing ensemble accuracy is more difficult.

To address the data gap, as well as dramatically simplify and accelerate RNA ensemble determination, we took advantage of structure prediction tools that leverage the growing database of RNA structures to directly generate a conformational library from a secondary structure that broadly samples energetically favorable $3 \mathrm{D}$ conformations. We used Fragment Assembly of RNA with Full-Atom Refinement (FARFAR) ${ }^{19}$, given its high performance in extensive tests of blind prediction of 3D RNA structure ${ }^{20}$. We then determined RNA ensembles by using NMR residual dipolar coupling (RDC) data ${ }^{21-23}$ to guide selection of conformers from the FARFAR library ${ }^{18,24,25}$.

We tested our approach on the transactivation response element (TAR) (Fig. 1a) from HIV-126,27, which has served as a model system for bulge motifs. Bulges are one of the most common RNA secondary structural elements that act as dynamic joints connecting helical elements, enabling their relative orientation to change adaptively during folding and function $^{18,26-28}$. We used FARFAR to directly generate a conformational library $(N=$ 10,000) from an input TAR secondary structure only constraining Watson-Crick base pairs (bps) inferred by NMR, and assuming an idealized A-form geometry ${ }^{29}$ for these bps while predicting the structure for all remaining nucleotides (Methods). We then tested and optimized the FARFAR-library using a rich dataset of four independent RDCs ( 8 RDCs per nucleotide) previously measured for four TAR molecules variably elongated to modulate alignment relative to the NMR magnetic field ${ }^{18,25}$. 

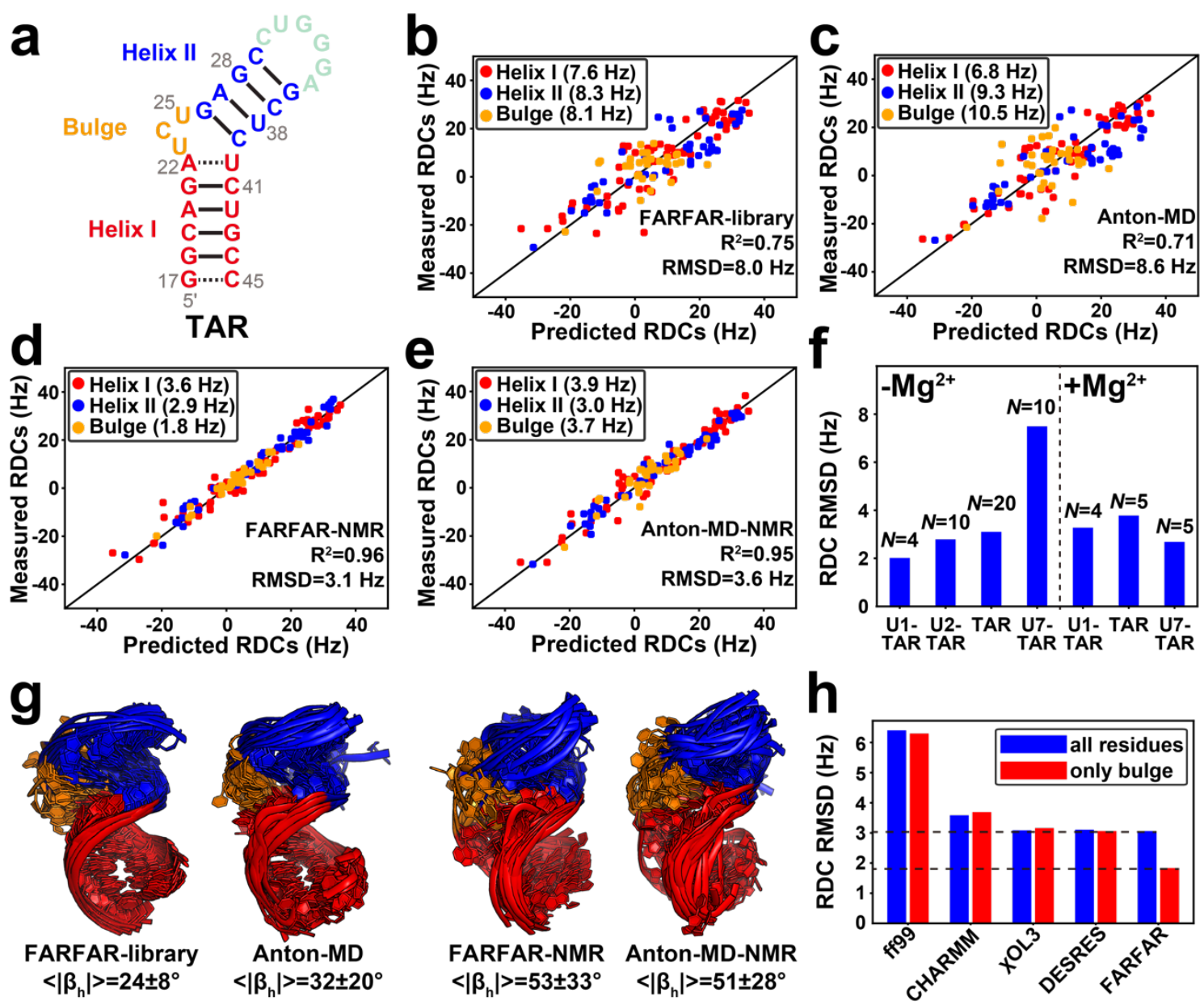

Fig. 1 Using FARFAR-NMR to determine a TAR ensemble. (a) Secondary structure of TAR. (b-e) Comparison between measured and predicted TAR RDCs for the (b) FARFAR-library $(N=10,000)$, (c) Anton-MD library $(N=10,000)$, (d) FARFAR-NMR $(N$ $=20)$ and (e) Anton-MD-NMR $(N=20)$. Values in parentheses denote the RDC RMSD. RDCs are color-coded according to the structural elements in Fig. 1a. (f) RDC RMSD for ensembles of TAR variants with ensemble size $N$ obtained using FARFAR-NMR. (g) Structural overlay of the TAR ensembles $(N=20)$ (Methods). $\quad\left|\beta_{\mathrm{h}}\right|$ is the absolute magnitude of the bend angle. (h) Comparison of RDC RMSD over all (blue) or bulge (red) residues for ensembles generated using different MD force fields (ff99 ${ }^{30}$, CHARMM36 ${ }^{31}$, ff99bsc0 $\chi O L 3^{32}$ and modified ff14 DESRES ${ }^{33}$ ) and FARFAR.

Intriguingly, the FARFAR-library showed better agreement (RMSD 8.0 versus 8.6

$\mathrm{Hz}$ ) with the RDCs (Fig. 1b) as compared to a previously reported ${ }^{18}$ TAR library (Anton- 
MD) generated by subjecting an experimentally determined NOE-based NMR structure of TAR (PDB ID 1ANR) ${ }^{26}$ to MD simulations with the CHARMM36 force field ${ }^{31}$ (Fig. 1c). An optimized ensemble with $N=20$ conformers (Extended Data Fig. 1b) was generated (Methods) by using the RDC agreement to guide selection of conformers from the FARFAR-library (Fig. 1d) ${ }^{18,24}$. The optimized FARFAR-NMR ensemble also better predicted the RDCs (RMSD 3.1 Hz) relative to the optimized Anton-MD-NMR ensemble (RMSD $3.6 \mathrm{~Hz}$ ) obtained using a similar procedure and the Anton-MD pool (Fig. 1e and Extended Data Fig. 1b). Cross validation ${ }^{18,34}$ showed that the improved RDC agreement was not due to over-fitting (Extended Data Fig. 1c). Similar RDC agreement (Fig. 1f) was obtained when using FARFAR-NMR to generate ensembles for TAR in the presence of $\mathrm{Mg}^{2+}$, and three additional TAR mutants ${ }^{28}$ containing one (U1-TAR), two (U2-TAR), and seven (U7-TAR) bulge nucleotides in the presence and absence of $\mathrm{Mg}^{2+}$ (Extended Data Fig. 2-3 and Supplementary Discussion), establishing the generality of the approach.

The improved agreement observed with the FARFAR-NMR ensemble was surprising given that the global inter-helical distribution ${ }^{35}$ of the Anton-MD-NMR TAR ensemble has been independently validated via X-ray scattering interferometry (XSI) ${ }^{36}$. Indeed, the FARFAR-NMR and Anton-MD-NMR ensembles show comparable agreement with the RDCs measured for helical bps (Fig. 1d, e) and the two ensembles sample similar inter-helical orientational distributions (Fig. 1g and Extended Data Fig. 4a-d).

Rather, the improved agreement was primarily driven by the RDCs measured in the locally more flexible bulge residues. For the FARFAR-NMR ensemble, the RDC RMSD 
(1.8 Hz) for bulge residues (Fig. 1d) was within experimental error $(\sim 2 \mathrm{~Hz})$, but was substantially higher $(3.7 \mathrm{~Hz})$ for the Anton-MD-NMR ensemble (Fig. 1e). The bulge RDC RMSD could not be improved by running MD simulations using different force fields (Fig. 1h), indicating that the improved conformational sampling in the FARFAR-library makes it possible to surpass the accuracy with which the TAR bulge could be described using conventional MD simulations. This improved performance is particularly noteworthy when considering that determining a high-resolution structure and running MD can take several months and often years whereas the FARFAR-library is generated within 24 hours running on 100 cores in parallel.

To further evaluate the accuracy of the FARFAR-NMR TAR ensemble, we substantially expanded the breadth and depth of atomic-level experimental data that can be brought to bear when evaluating the accuracy of RNA ensembles by predicting ensembleaveraged ${ }^{1} \mathrm{H},{ }^{13} \mathrm{C}$, and ${ }^{15} \mathrm{~N}$ chemical shifts ( $\sim 15$ chemical shifts per nucleotide) using quantum-mechanical AF-QM/MM calculations ${ }^{37,38}$. Although rich in structural information that is complimentary to that obtained from RDCs, the agreement between measured chemical shifts and values predicted from crystal structures of nucleic acids has traditionally been poor ${ }^{37,39}$. We recently showed in studies of DNA duplexes that at least some of this disagreement originates from neglecting ensemble averaging when predicting ${ }^{13} \mathrm{C}$ chemical shifts ${ }^{38}$. This revelation and the improved FARFAR-NMR TAR ensemble led us to test this approach on flexible RNAs, and to extend its reach by incorporating ${ }^{1} \mathrm{H}$ and ${ }^{15} \mathrm{~N}$ shifts in addition to ${ }^{13} \mathrm{C}$ chemical shifts (Fig. 2a). 
a

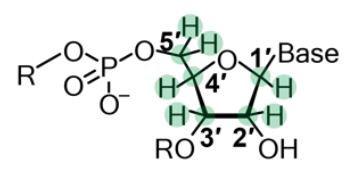

b
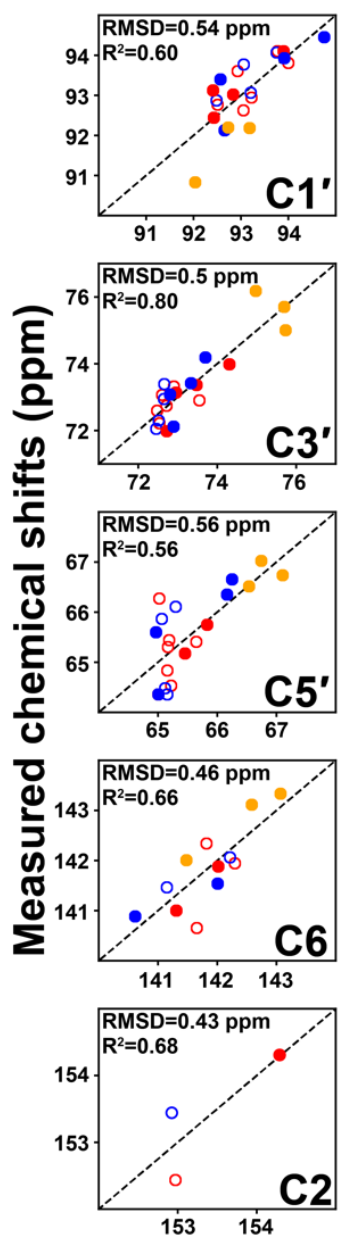

C2

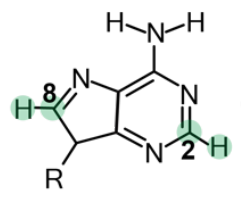

Adenine<smiles></smiles>

Guanine<smiles></smiles>

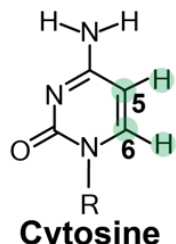

C
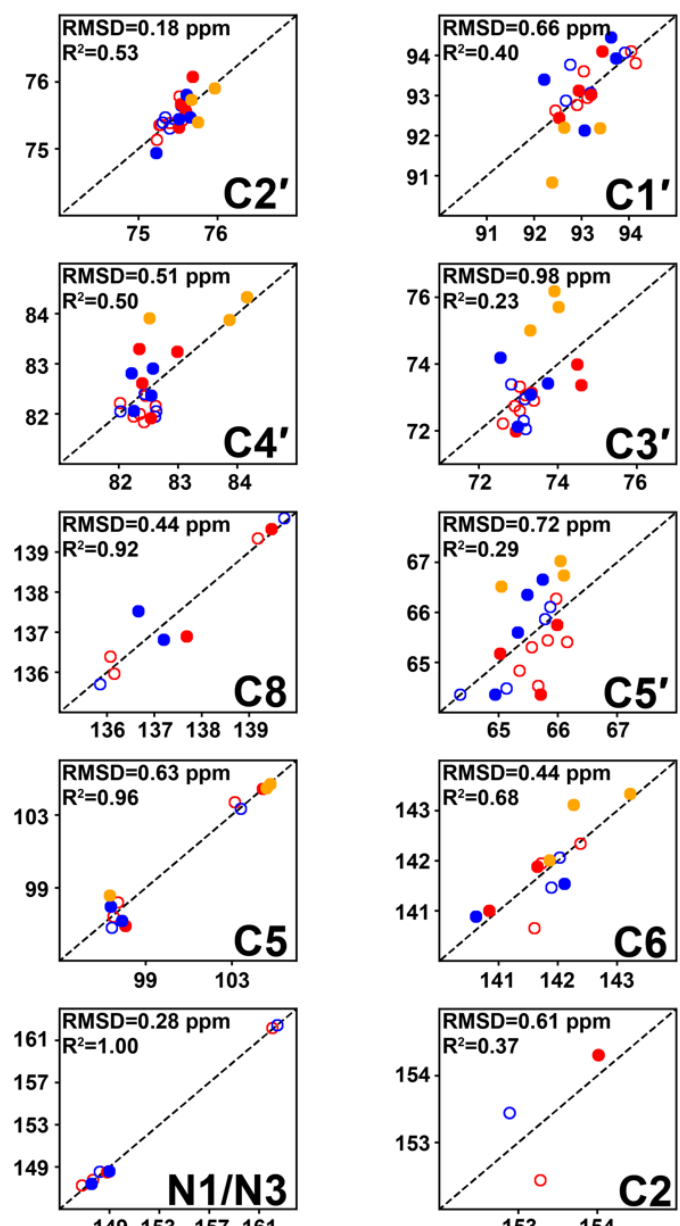

149153157161 Predicted chemical shifts (ppm)
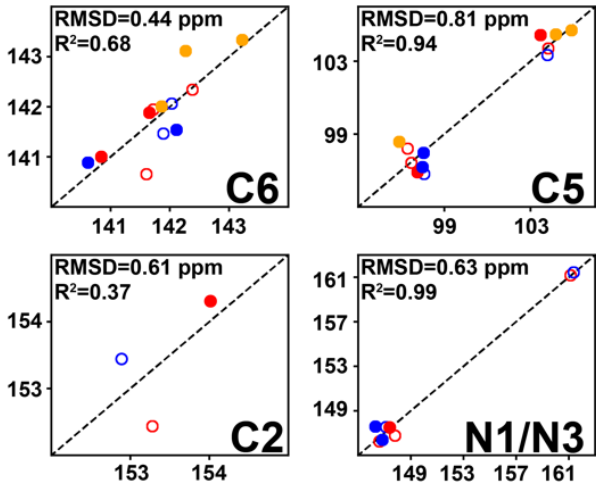

$149 \begin{array}{lll}153 & 157161\end{array}$
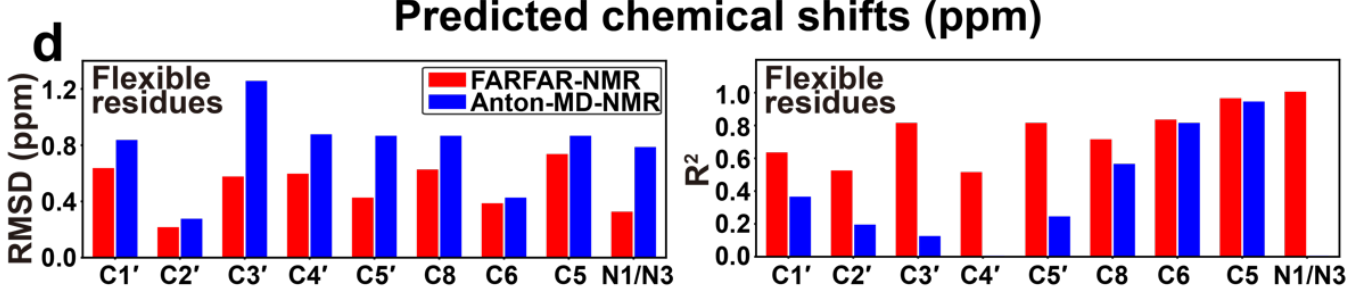

Fig. 2 Evaluating TAR ensembles using chemical shifts. (a) Chemical structures of the sugar and base moieties with chemical shift probes used to test ensemble accuracy highlighted in green. (b-c) Comparison of measured and predicted ${ }^{13} \mathrm{C} /{ }^{15} \mathrm{~N}$ chemical shifts for (b) FARFAR-NMR $(N=20)$ (c) Anton-MD-NMR $(N=20)$ by AF-QM/MM 
(Methods). Values are color-coded according to the structural elements in Fig. 1a. Chemical shifts for central Watson-Crick bps within A-form helices (C19-G43, A20-U42, G21-C41, A27-U38, G28-C37) are denoted using open circles. A correction was applied to the predicted chemical shifts (Methods) as described previously ${ }^{38}$. For ${ }^{1} \mathrm{H}$ chemical shifts see Extended Data Fig. 5b, d. (d) Comparison of RMSD (left) and $\mathrm{R}^{2}$ (right) between measured and predicted ${ }^{13} \mathrm{C} /{ }^{15} \mathrm{~N}$ chemical shifts for flexible residues (U23, C24, U25, A22-U40, G26-C39, C29-G36, G18-C44) for FARFAR-NMR (red) and Anton-MDNMR (blue).

Remarkably, good agreement was observed between the measured ${ }^{1} \mathrm{H},{ }^{13} \mathrm{C}$, and ${ }^{15} \mathrm{~N}$ base and sugar chemical shifts and values back-calculated for the FARFAR-library (Extended Data Fig. 5 and Supplementary Discussion). The agreement improved substantially for the FARFAR-NMR ensemble following RDC optimization for $48 \%$ of the atom-types analyzed, while the agreement was unaffected for the remaining atom-types (Fig. 2b and Extended Data Fig. 5). The agreement deteriorated substantially for any one conformer member of the ensemble, underscoring the critical importance of ensembleaveraging (Extended Data Fig. 6). The agreement with an independent set of measurements not used in ensemble determination that is highly sensitive to various structural features of the base, sugar, and backbone strongly suggests that FARFAR-NMR describes the TAR ensemble with atomic accuracy ( $<2 \AA$, Methods). In sharp contrast, the agreement was much weaker for the Anton-MD-NMR ensemble relative to FARFARNMR for $90 \%$ of the atom-types, particularly for sugar chemical shifts, some of which show no apparent correlation even following RDC optimization (Fig. 2c, $d$ and Extended Data Fig. 5). These results establish the utility of chemical shifts in RNA ensemble determination and also show that the improved accuracy of the FARFAR-NMR ensemble 
relative to Anton-MD-NMR is even greater than alluded to by the RDC data.

We compared the FARFAR-NMR and Anton-MD-NMR ensembles (Fig. 3a) to better understand the features responsible for the more accurate ensemble description of the TAR bulge. Approximately $75 \%$ of the conformers in the FARFAR-NMR ensemble have the canonical UCU bulge, while the remaining 25\% have a non-canonical AUC bulge that forms through a single nucleotide register shift (Extended Data Fig. 7a). In contrast, conformers in the Anton-MD-NMR ensemble sample a broader set of junction topologies some of which diverge from the NMR-derived Watson-Crick pairing (Extended Data Fig. $7 \mathrm{~b}$ and Supplementary Discussion), and this could contribute to the poor agreement observed with the imino ${ }^{15} \mathrm{~N} /{ }^{1} \mathrm{H}$ chemical shifts (Fig. 2c and Extended Data Fig. 5). 
a

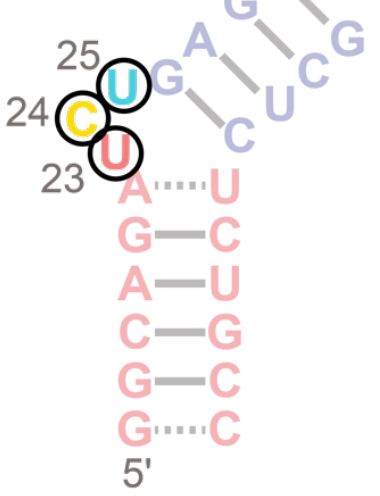

FARFAR-NMR

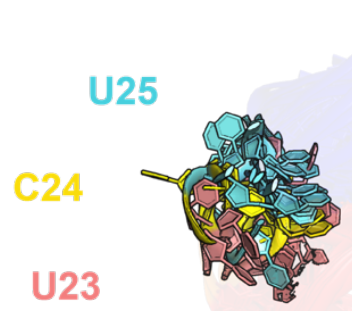

Anton-MD-NMR

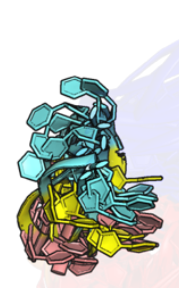

b

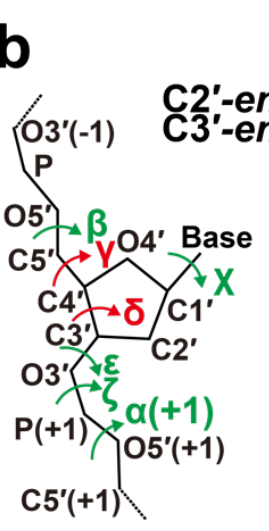

C U23

C24

U25

A22

U40
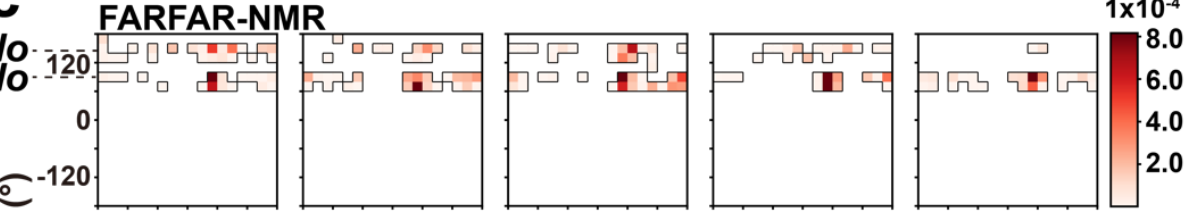

Anton-MD-NMR
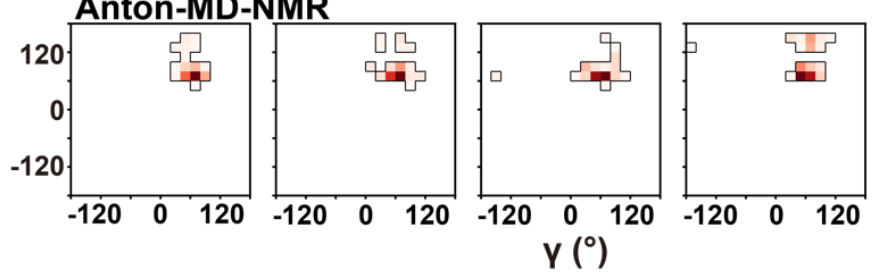

$1 \times 10^{-4}$

d C3'-endo

e
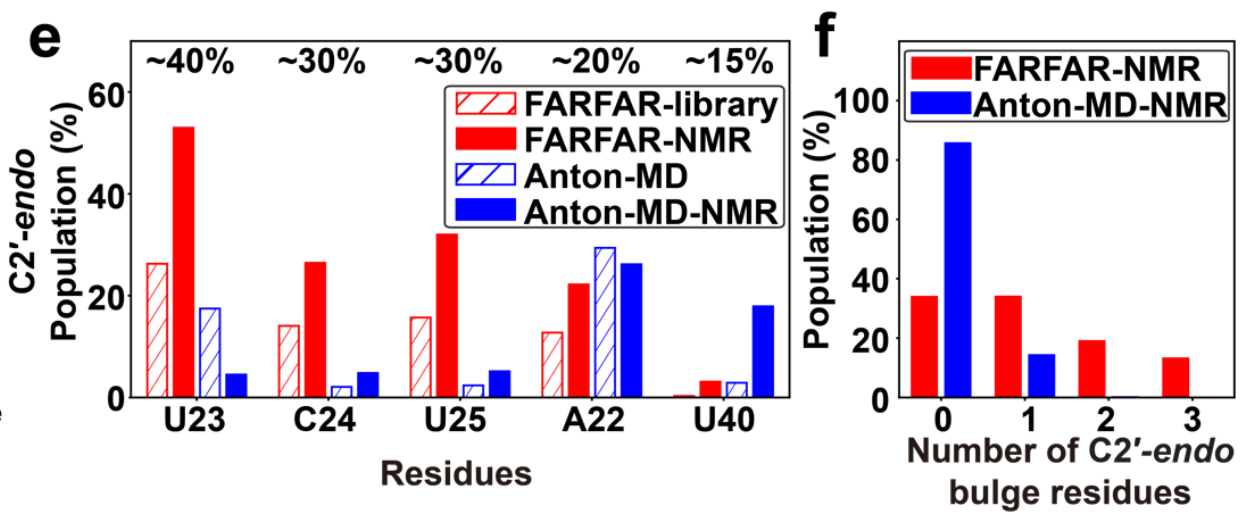

Fig. 3 FARFAR-NMR ensemble more broadly samples non-canonical sugar-backbone torsion angles relative to Anton-MD-NMR. (a) Overlay of the dynamic ensembles of the TAR bulge.

(b) RNA backbone torsion angles exhibiting different and similar distributions between Anton-MD-NMR and FARFAR-NMR are colored red and green, respectively (Methods). (c) 2D density maps of $\delta$ versus $\gamma$ comparing Anton-MD-NMR and FARFAR-NMR ensembles $(N=2,000)$ for bulge residues as well as A22 and U40. The bin width is $20^{\circ}$. (d) Structure of the ribose moiety in $\mathrm{C} 3^{\prime}$-endo and $\mathrm{C} 2^{\prime}$-endo conformations. (e) Population of $\mathrm{C} 2^{\prime}$-endo pucker at bulge residues as well as A22 and U40 in the FARFAR-library $(N=10,000$, red open), FARFAR-NMR $(N=2,000$, red fill), Anton-MD library $(N=10,000$, blue open) and Anton-MD-NMR $(N=2,000$, blue fill). 
Experimental estimates of the $\mathrm{C} 2$ '-endo population based on ${ }^{13} \mathrm{C}$ chemical shifts are indicated above the bars (Methods). (f) The population of conformers in the ensemble as a function of the number of C2'-endo bulge residues for FARFAR-NMR (red, $N=2,000$ ) and Anton-MD-NMR (blue, $N=2,000$ ).

In addition, despite having a narrower range of junction topologies, the sugar pucker distribution (defined by angle $\delta$ ) for bulge residues in the FARFAR-NMR sample a broader range (Fig. 3b, c), are substantially enriched in the non-canonical $\mathrm{C}^{\prime}$-endo conformation (Fig. 3d) relative to the Anton-MD-NMR ensemble, and are in better agreement with the NMR-derived ${ }^{40}$ sugar puckers (Fig. 3e and Extended Data Fig. 8a). Moreover, $\sim 15 \%$ of the conformers in the FARFAR-NMR ensemble had all three bulge residues simultaneously in the $\mathrm{C} 2^{\prime}$-endo conformation but none did in the Anton-MD-NMR ensemble (Fig. 3f). Excluding all conformers containing $\mathrm{C}^{\prime}$ '-endo sugar puckers in U23, C24, U25, A22 or U40 from the FARFAR-library diminished the RDC agreement to a level similar to the Anton-MD derived ensembles again confirming that the improved agreement is not due to over-fitting of the data (Extended Data Fig. 8b, c). A similar behavior was observed for the backbone torsion angle $\gamma$ (Fig. 3b), which shows greater sampling of nongauche+ angles in the FARFAR relative to the Anton-derived ensembles (Fig. 3c and Extended Data Fig. 8b, d). This can account for the better agreement observed for sugar chemical shifts ${ }^{40}$ in the FARFAR-NMR versus Anton-MD-NMR ensembles (Fig. 2b-d).

What causes conformers in the FARFAR-NMR ensemble to more greatly sample non-canonical sugar-backbone conformations at the bulge relative to conformers in the Anton-MD-NMR ensemble? Unpaired pyrimidine RNA nucleotides unconstrained by 
other interactions are enriched in the $\mathrm{C}^{2}$-endo conformation $\left(\mathrm{C}^{\prime}\right.$-endo: $\mathrm{C} 3$ '-endo is 40:60) ${ }^{41}$ and C3'-endo becomes the predominant sugar-pucker when the nucleotides form bps or stack intra-helically ${ }^{40}$. Indeed, $\sim 80 \%$ of the residues with $\mathrm{C}^{\prime}$-endo sugar puckers were extra-helical in the FARFAR-NMR ensemble (Fig. 4a and Extended Data Fig. 7a). Moreover, for linear inter-helical conformations, there was a strong preference to have all three bulge residues simultaneously flip out (Fig. 4b) and adopt conformations enriched in the $\mathrm{C} 2$ '-endo sugar pucker (Fig. 4c); the bulge residues flip out to allow the two helices to coaxially stack (Supplementary Video 1 and Extended Data Fig. 7a). Such coaxial conformations have previously been hypothesized to exist within the TAR ensemble based on the $\mathrm{Mg}^{2+}$ dependence of the inter-helical ensemble ${ }^{28}$ and a crystal structure of $\mathrm{Ca}^{2+}$ bound $\mathrm{TAR}^{42}$. 
a

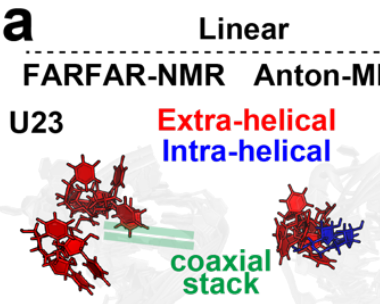

\section{C24}
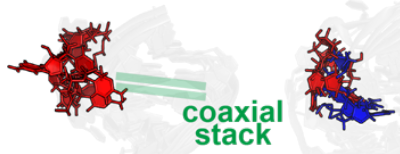

U25

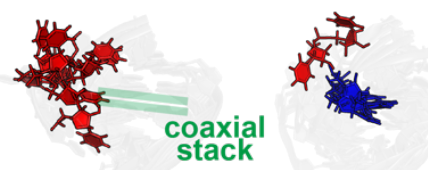

b $\begin{aligned} & \text { Number of extra-helical } \\ & \text { bulge residues }\end{aligned}$

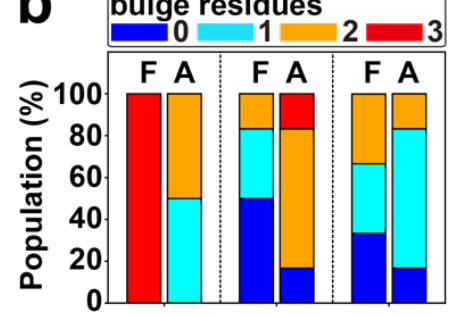

linear bend kinked

\section{C}

Number of C2'-endo

C bulge residues

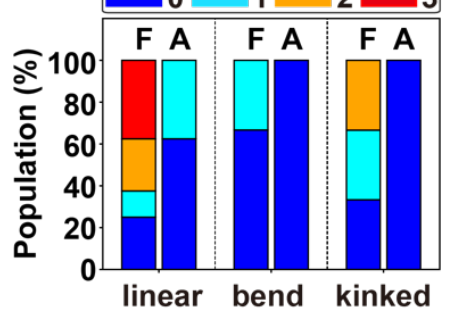

Intermediate bend

FARFAR-NMR Anton-MD-NMR
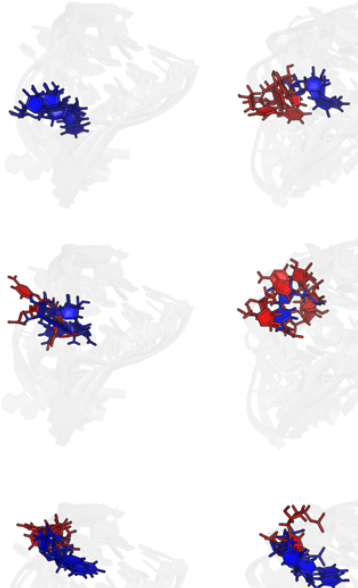

d

FARFAR-NMR $\mathrm{TAR}+\mathrm{Ca}^{2+}$

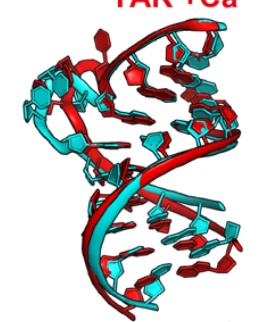

RMSD=1.4 A

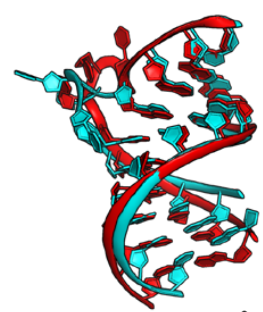

RMSD=1.3 A
Kinked

FARFAR-NMR Anton-MD-NMR
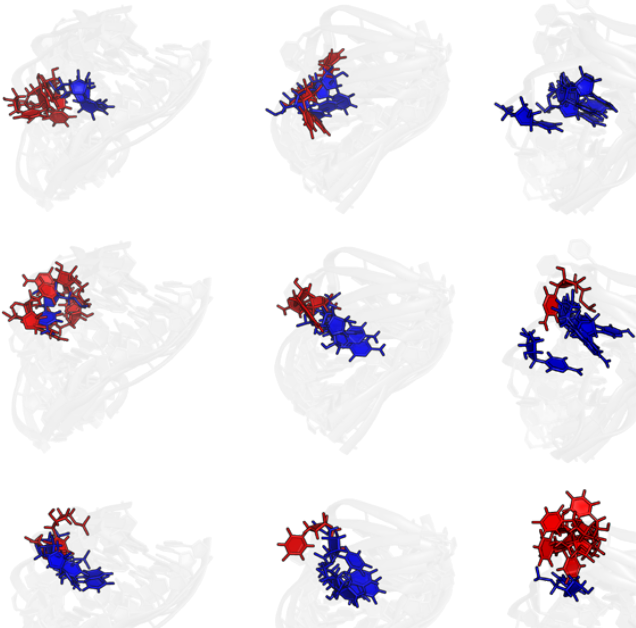

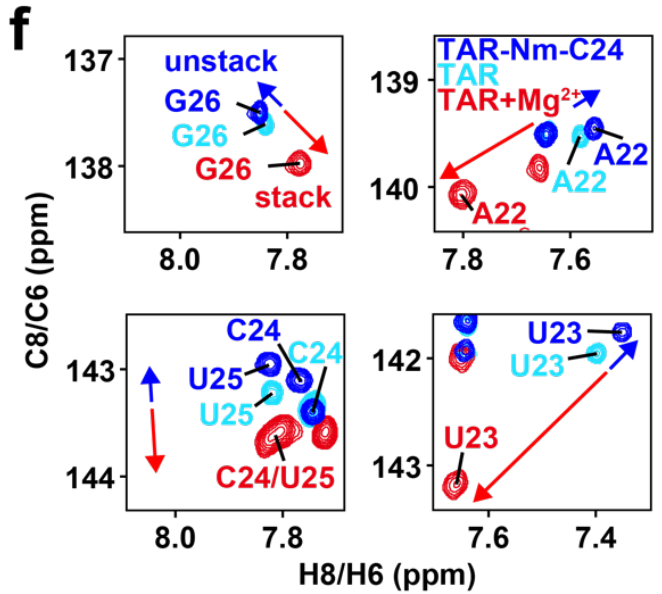

Fig. 4 Cooperative extra-helical flipping and sugar repuckering of bulge residues are coupled to coaxial stacking. (a) Overlay of conformers showing motions in bulge residues for linear $\left(\left|\beta_{\mathrm{h}}\right|<45^{\circ}\right)$, intermediate bend $\left(45^{\circ}<\left|\beta_{\mathrm{h}}\right|<70^{\circ}\right)$ and kinked $\left(\left|\beta_{\mathrm{h}}\right|>70^{\circ}\right)$ inter-helical conformations in the FARFAR-NMR and Anton-MD-NMR ensembles. (bc) The fractional populations of conformers with (b) extra-helical and (c) C2'-endo bulge residues (color-coded) as a function of bending angle in the FARFAR-NMR and AntonMD-NMR ensembles. $\quad F$ and $A$ denote FARFAR-NMR and Anton-MD-NMR, respectively. (d) Comparison of two coaxially stacked FARFAR-NMR conformers with the crystal structure of $\mathrm{Ca}^{2+}$ bound TAR (PDB: 397D) ${ }^{42}$. (e) Nm shifts sugar pucker equilibrium towards $\mathrm{C} 3$ '-endo. (f) Overlay of $2 \mathrm{D}\left[{ }^{13} \mathrm{C},{ }^{1} \mathrm{H}\right] \mathrm{NMR}$ HSQC spectra of the 
aromatic spins for TAR-Nm-C24 without $\mathrm{Mg}^{2+}$ (blue, inducing unstacking), TAR without $\mathrm{Mg}^{2+}$ (cyan) and TAR with $\mathrm{Mg}^{2+}$ (red, inducing stacking).

Strikingly, two of the coaxial conformations in the FARFAR-NMR ensemble superimpose with the TAR crystal structure with a heavy-atom RMSD of 1.3-1.4 $\AA$ (Fig. 4d). Thus, the TAR crystal structure captures substates of the ensemble in solution. In sharp contrast, none of the conformations in the Anton-MD-NMR ensemble had the two helices coaxially stacked, and not a single conformer had all three bulge residues flipped out and having the $\mathrm{C}^{\prime}$-endo sugar pucker (Fig. 4a-c and Extended Data Fig. 7b). The FARFAR-NMR ensemble (Fig. 4a and Supplementary Video 1) further shows that all three bulge residues simultaneously flip out with high cooperativity $(\sim 2.3 \mathrm{kcal} / \mathrm{mol}$, see methods) most likely because this then permits favorable coaxial stacking of the helices (Fig. 4a, Extended Data Fig. 7a and Supplementary Video 1). This can explain prior results ${ }^{43}$ showing that a TAR mutation that promotes inter-helical stacking results in a predominantly coaxially stacked conformation in which all three bulge residues are flipped out.

Finally, we put key atomic features of the FARFAR-NMR ensemble to a test by rationally redistributing the conformer populations using atomic mutagenesis. The ensemble shows that the kinked unstacked conformations are enriched in bulge residues that have the $\mathrm{C}^{\prime}$ '-endo sugar pucker relative to the coaxial stacked conformations (Fig. 4c). To test this feature of the ensemble, we incorporated 2'-O-Methyl (Nm) modifications (Fig. 4e) at $\mathrm{U} 23$ or $\mathrm{C} 24$ to bias the sugar pucker at these positions toward C3'-endo by $\sim 0.2$ 
$\mathrm{kcal} / \mathrm{mol}^{44}$. Indeed, methylating either $\mathrm{U} 23$ or C24 resulted in chemical shift perturbations in 2D NMR spectra of TAR (Fig. 4f and Extended Data Fig. 9) throughout the bulge and neighboring residues that are specifically directed towards the chemical shifts of the unstacked conformation ${ }^{28}$, as expected for a cooperative redistribution in favor of the kinked conformation.

In conclusion, FARFAR-NMR lays the foundation for a new paradigm for RNA ensemble determination by combining reliably measurable NMR data with $3 \mathrm{D}$ structure prediction. The approach can immediately be applied to render many existing NMR structures of RNAs that were determined using conventional approaches into dynamic ensembles, and their accuracies can be tested using the available chemical shift data. The approach is general, rapid, and can also incorporate other sources of experimental data. Given its ease of implementation and higher throughput, FARFAR-NMR has the potential to unleash the ensemble description of RNAs to all corners of biology, from which a deeper and broader understanding of folding and function will undoubtedly emerge. 


\section{Main references}

1 Frauenfelder, H., Sligar, S.G., \& Wolynes, P.G., The energy landscapes and motions of proteins. Science 254 (5038), 1598-1603. (1991).

2 Jensen, M.R. et al., Quantitative determination of the conformational properties of partially folded and intrinsically disordered proteins using NMR dipolar couplings. Structure 17 (9), 1169-1185 (2009).

3 van den Bedem, H. \& Fraser, J.S., Integrative, dynamic structural biology at atomic resolution--it's about time. Nat. Methods 12 (4), 307-318 (2015).

4 Ganser, L.R., Kelly, M.L., Herschlag, D., \& Al-Hashimi, H.M., The roles of structural dynamics in the cellular functions of RNAs. Nat. Rev. Mol. Cell Biol. 20 (8), 474-489 (2019).

5 Breaker, R.R., Prospects for riboswitch discovery and analysis. Mol. Cell 43 (6), 867-879 (2011).

6 Stagno, J.R. et al., Structures of riboswitch RNA reaction states by mix-and-inject XFEL serial crystallography. Nature 541 (7636), 242-246 (2017).

7 Jarmoskaite, I. et al., A Quantitative and Predictive Model for RNA Binding by Human Pumilio Proteins. Mol. Cell 74 (5), 966-981.e918 (2019).

$8 \quad$ Harcourt, E.M., Kietrys, A.M., \& Kool, E.T., Chemical and structural effects of base modifications in messenger RNA. Nature 541 (7637), 339-346 (2017).

9 Halvorsen, M., Martin, J.S., Broadaway, S., \& Laederach, A., Disease-associated mutations that alter the RNA structural ensemble. PLoS Genet. 6 (8), e1001074 (2010).

10 Steinert, H. et al., Pausing guides RNA folding to populate transiently stable RNA structures for riboswitch-based transcription regulation. Elife 6 (2017).

11 Watters, K.E., Strobel, E.J., Yu, A.M., Lis, J.T., \& Lucks, J.B., Cotranscriptional folding of a riboswitch at nucleotide resolution. Nat. Struct. Mol. Biol. 23 (12), 1124-1131 (2016).

12 Larsen, K.P., Choi, J., Prabhakar, A., Puglisi, E.V., \& Puglisi, J.D., Relating Structure and Dynamics in RNA Biology. Cold Spring Harb. Perspect. Biol. 11 (7) (2019).

13 Choy, W.Y. \& Forman-Kay, J.D., Calculation of ensembles of structures representing the unfolded state of an SH3 domain. J. Mol. Biol. 308 (5), 1011-1032 (2001).

14 Ozenne, V. et al., Mapping the Potential Energy Landscape of Intrinsically Disordered Proteins at Amino Acid Resolution. J. Am. Chem. Soc. 134 (36), 1513815148 (2012).

15 Lange, O.F. et al., Recognition dynamics up to microseconds revealed from an RDC-derived ubiquitin ensemble in solution. Science 320 (5882), 1471-1475 (2008). 
Showalter, S.A. \& Brüschweiler, R., Quantitative molecular ensemble interpretation of NMR dipolar couplings without restraints. J. Am. Chem. Soc. 129 (14), 4158-4159 (2007). Sponer, J. et al., RNA Structural Dynamics As Captured by Molecular Simulations: A Comprehensive Overview. Chem. Rev. 118 (8), 4177-4338 (2018). Salmon, L., Bascom, G., Andricioaei, I., \& Al-Hashimi, H.M., A General Method for Constructing Atomic-Resolution RNA Ensembles using NMR Residual Dipolar Couplings: The Basis for Interhelical Motions Revealed. J. Am. Chem. Soc. 135 (14), 5457-5466 (2013). Watkins, A.M. \& Das, R., FARFAR2: Improved de novo Rosetta prediction of complex global RNA folds. bioRxiv, 764449 (2019). Miao, Z. et al., RNA-Puzzles Round III: 3D RNA structure prediction of five riboswitches and one ribozyme. RNA 23 (5), 655-672 (2017).

21 Tjandra, N. \& Bax, A., Direct measurement of distances and angles in biomolecules by NMR in a dilute liquid crystalline medium. Science 278 (5340), 1111-1114 (1997).

22 Tolman, J.R., Flanagan, J.M., Kennedy, M.A., \& Prestegard, J.H., Nuclear Magnetic Dipole Interactions in Field-Oriented Proteins - Information For Structure Determination in Solution. Proc. Natl. Acad. Sci. U.S.A. 92 (20), 92799283 (1995).

23 Schwieters, C.D. \& Clore, G.M., A physical picture of atomic motions within the Dickerson DNA dodecamer in solution derived from joint ensemble refinement against NMR and large-angle X-ray scattering data. Biochemistry 46 (5), 11521166 (2007). Chen, Y., Campbell, S.L., \& Dokholyan, N.V., Deciphering protein dynamics from NMR data using explicit structure sampling and selection. Biophys. J. 93 (7), 23002306 (2007). correlated dynamics that directs RNA conformational transitions. Nature 450 (7173), 1263-1267 (2007). of ligands reveals a novel conformation of the trinucleotide bulge. Nucleic Acids Res. 24 (20), 3974-3981 (1996).

27 Puglisi, J.D., Tan, R., Calnan, B.J., Frankel, A.D., \& Williamson, J.R., Conformation of the TAR RNA-arginine complex by NMR spectroscopy. Science 257, 76-80 (1992).

Merriman, D.K. et al., Increasing the length of poly-pyrimidine bulges broadens RNA conformational ensembles with minimal impact on stacking energetics. $R N A$ 24 (10), 1363-1376 (2018). 
Musselman, C. et al., Impact of static and dynamic A-form heterogeneity on the determination of RNA global structural dynamics using NMR residual dipolar couplings. J. Biomol. NMR 36 (4), 235-249 (2006). Cornell et al. force field with improved sugar pucker phases and helical repeat. $J$. Biomol. Struct. Dyn. 16 (4), 845-862 (1999). Denning, E.J., Priyakumar, U.D., Nilsson, L., \& Mackerell, A.D., Jr., Impact of 2'hydroxyl sampling on the conformational properties of RNA: update of the CHARMM all-atom additive force field for RNA. J. Comput. Chem. 32 (9), 19291943 (2011).

32 Zgarbova, M. et al., Refinement of the Cornell et al. Nucleic Acids Force Field Based on Reference Quantum Chemical Calculations of Glycosidic Torsion Profiles. J. Chem. Theory Comput. 7 (9), 2886-2902 (2011). comparable to state-of-the-art protein force fields. Proc. Natl. Acad. Sci. U.S.A. 115 (7), E1346-e1355 (2018).

Salmon, L., Yang, S., \& Al-Hashimi, H.M., Advances in the determination of nucleic acid conformational ensembles. Annu. Rev. Phys. Chem. 65, 293-316 (2014).

Bailor, M.H., Mustoe, A.M., Brooks, C.L., 3rd, \& Al-Hashimi, H.M., 3D maps of RNA interhelical junctions. Nat. Protoc. 6 (10), 1536-1545 (2011).

Shi, X., Walker, P., Harbury, P.B., \& Herschlag, D., Determination of the conformational ensemble of the TAR RNA by X-ray scattering interferometry. Nucleic Acids Res. 45 (8), e64 (2017).

37 Swails, J., Zhu, T., He, X., \& Case, D.A., AFNMR: automated fragmentation quantum mechanical calculation of NMR chemical shifts for biomolecules. $J$. Biomol. NMR 63 (2), 125-139 (2015).

Shi, H. et al., Atomic structures of excited state A-T Hoogsteen base pairs in duplex DNA by combining NMR relaxation dispersion, mutagenesis, and chemical shift calculations. J. Biomol. NMR 70 (4), 229-244 (2018).

Case, D.A., Chemical shifts in biomolecules. Curr. Opin. Struct. Biol. 23 (2), 172176 (2013).

40 Clay, M.C., Ganser, L.R., Merriman, D.K., \& Al-Hashimi, H.M., Resolving sugar puckers in RNA excited states exposes slow modes of repuckering dynamics. Nucleic Acids Res. 45 (14), e134 (2017).

41 Davies, D.B., Conformations of nucleosides and nucleotides. Prog. Nucl. Magn. Reson. Spectrosc. 12 (3), 135-225 (1978).

42 Ippolito, J.A. \& Steitz, T.A., A 1.3-angstrom resolution crystal structure of the HIV-1 trans- activation response region RNA stem reveals a metal ion- dependent bulge conformation. Proc. Natl. Acad. Sci. U.S.A. 95 (17), 9819-9824 (1998). 
43 Stelzer, A.C., Kratz, J.D., Zhang, Q., \& Al-Hashimi, H.M., RNA Dynamics by Design: Biasing Ensembles Towards the Ligand-Bound State. Angew. Chem. Int. Ed. Engl. 49(33), 5731-5733 (2010).

44 Kawai, G. et al., Conformational rigidity of specific pyrimidine residues in tRNA arises from posttranscriptional modifications that enhance steric interaction between the base and the 2'-hydroxyl group. Biochemistry 31 (4), 1040-1046 (1992). 


\section{Methods}

\section{Generating ensembles using FARFAR}

FARFAR $^{19}$ is implemented as the rna_denovo program in Rosetta Software Suite. FARFAR requires as input the RNA sequence, which can be constrained by an optional secondary structure. The input secondary structures for TAR were derived based on the imino ${ }^{1} \mathrm{H}$ resonances in NMR spectra. Therefore, Watson-Crick base pairing was imposed for G18-C44, C19-G43, A20-U42, G21-C41, G26-C39, A27-U38, G28-C37 and C29-G36 whereas nucleotides in the apical loop and bulge, and the terminal G17-C45 bp were set to be unconstrained. Given the indirect NMR observation of the A22-U40 bp in U2-TAR by H6(C5)NN experiment as reported previously ${ }^{28}$, two sets of FARFAR simulations were conducted for each TAR variant with A22-U40 either paired or unpaired. Interestingly, with the exception of U2-TAR, constraining A22-U40 when generating FARFAR-libraries did not improve the agreement with the RDCs for the TAR and other variants. This highlights the importance of differentiating between well-formed versus labile bps, an important advantage offered by NMR as compared to other methods for determining secondary structure such as chemical probing. Thus, all the FARFARlibraries in this study correspond to those in which the A22-U40 base pair is not constrained, except for the U2-TAR ensemble in the absence of $\mathrm{Mg}^{2+}$. To significantly reduce the run time, we modeled the helices of TAR and its bulge variants as static idealized A-form helices shown previously to satisfy NMR RDC data in $\mathrm{RNA}^{28,29}$. rna_helix.py is a python 
wrapper for the Rosetta executable rna_helix. rna_helix.py is available in \$ROSETTA/tools/rna_tools/bin, where \$ROSETTA is the Rosetta installation path.

$$
\begin{aligned}
& \text { rna_helix.py -seq gcag cugc -resnum 18-21 41-44 -o helixl.pdb } \\
& \text { rna_helix.py -seq gagc gcuc -resnum 26-29 36-39 -o helix2.pdb }
\end{aligned}
$$

in which RNA sequences of both strands as well as their residue indices are required as input. While generating FARFAR-library assuming idealized A-form helices, we then executed rna_denovo with the following command:

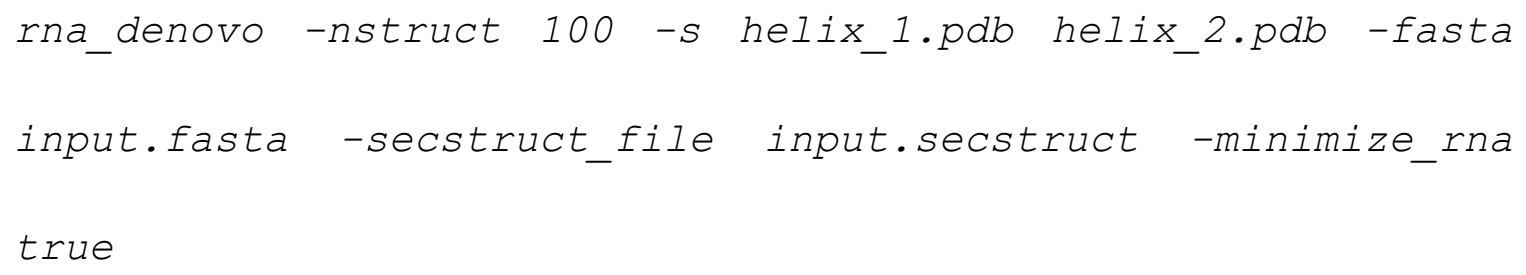

where -nstruct is the number of models per run which is 100 in the current study, fasta is the path of a fasta file containing the RNA sequence, -secstruct is the path of a file (input.secstruct) containing the RNA secondary structure in dot-bracket notation, -minimize_rna true minimizes the RNA after fragment assembly, and -s specifies the path to the pdb files that contain static structures of our helices we do not wish to generate via fragment assembly to save computation (the fasta, secstruct files as well as example commands can be found in Supplementary Table 2-5). 
The entire procedure was repeated 100-200 times and 10,000 structures were randomly selected from the entire resulting output with Rosetta energy units $<0$ to remove models that potentially may have chain breaks and severe steric clash which do not satisfy the RDCs (Extended Data Fig. 1a), to generate the FARFAR-library $(N=10,000)$.

\section{Molecular dynamics (MD) simulations}

Simulations of HIV-1 TAR starting from the PDB structure 1ANR using the CHARMM36 force field ${ }^{31}$ were performed as described previously ${ }^{18}$. MD simulations of TAR starting from the PDB structure 1ANR using the ff99 force field ${ }^{30}$ with and without ¿oL3 corrections for $\mathrm{RNA}^{32}$ were performed using periodic boundary conditions as implemented in the AMBER MD simulation package ${ }^{45}$. All starting structures were solvated using a truncated octahedral box of TIP $3 \mathrm{P}^{46}$ water molecules with box size chosen such that the boundary was at least $11 \AA$ away from any of the RNA atoms for all simulations with the ff 99 force field with $\chi_{\mathrm{OL} 3}$ corrections. For the TAR simulations with the ff99 force field without $\chi_{0 L 3}$ corrections, the system was solvated in a truncated octahedral box of SPC/E water molecules such that the boundary was at least $15.4 \AA$ away from any of the RNA atoms. $\mathrm{Na}^{+}$ions treated using the Joung-Cheatham parameters ${ }^{47}$ were then added to neutralize the charge of the system in all cases. The system was then energy minimized in two stages with the solute being fixed (with a restraint of 500 $\left.\mathrm{kcal} / \mathrm{mol} / \AA^{2}\right)$ during the first stage. Equilibration and production runs $(1 \mu \mathrm{s})$ were then performed as described previously ${ }^{48}$. Simulations of TAR starting from PDB 1ANR 
using the DESRES force field ${ }^{33}$ were performed using the GROMACS MD simulation package $^{49,50}$. DESRES force field files for GROMACS were obtained from a port by Giovanni Bussi (https://github.com/srnas/ff/tree/desres). The starting structure (PDB 1ANR) was solvated using a rhombic dodecahedral box of TIP4P-D ${ }^{51}$ water molecules, with box size chosen such that the boundary was at least $10 \AA$ away from any of the RNA atoms. $\mathrm{Na}^{+}$ions treated using the parameters from MacKerell et al. ${ }^{52}$ were then added to neutralize the charge of the system. After energy minimization without restraints, the system was then gradually heated to a temperature of to $298 \mathrm{~K}$ using a modified Berendsen thermostat with a stochastic term ${ }^{53,54}(\tau=0.1 \mathrm{ps})$, under constant volume conditions for $100 \mathrm{ps}$ with harmonic restraints on the solute $\left(1000 \mathrm{~kJ} / \mathrm{mol} / \mathrm{nm}^{2}\right)$. The system was then allowed to equilibrate for 100 ps under constant pressure (1 bar), using the ParinelloRahman barostat ${ }^{55,56}(\tau=2 \mathrm{ps})$ and temperature (at $298 \mathrm{~K}$, using a modified Berensen thermostat, $\tau=0.1 \mathrm{ps})$ conditions, with harmonic restraints on the solute $\left(1000 \mathrm{~kJ} / \mathrm{mol} / \mathrm{nm}^{2}\right)$. This was followed by NPT equilibration for $30 \mathrm{~ns}$ without harmonic restraints, following by a production run of $1 \mu \mathrm{s}$. A non-bonded cutoff of $9 \AA$ was used for treating short range non-bonded interactions while the Particle Mesh Ewald method ${ }^{57}$ was used to treat long range electrostatic interactions. Covalent bonds involving hydrogen were constrained using the LINCS algorithm ${ }^{58}$ to enable the use of a 2 fs timestep. A set of evenly ( 5 ps) spaced snapshots was used for subsequent analysis of all simulations using the CPPTRAJ suite of programs ${ }^{59}$. 
Additional MD simulations using the ff99 force field with $\chi_{\mathrm{OL} 3}$ corrections were also performed to assess the extent to which sampling of sugar puckers in the bulge could be influenced by changes in the starting structures used for the simulations (Extended Data Fig. 10). Two starting structures were derived from PDB 1ANR with the sugar puckers of $\mathrm{U} 23\left(\mathrm{TAR}^{\mathrm{U} 23 \mathrm{C}^{\prime} \text {-endo }}\right)$ and $\mathrm{U} 25\left(\mathrm{TAR}^{\mathrm{U} 25 \mathrm{C}^{\prime} \text {-endo }}\right)$ individually switched from $\mathrm{C}^{\prime}$-endo to $\mathrm{C} 2$ '-endo, and another was a conformer from the FARFAR-NMR ensemble (TAR ${ }^{\text {FARFAR }}$ ) in which the sugar puckers of U23, C24 and U25 were all $\mathrm{C}^{\prime}$ '-endo. The starting structures for the $\mathrm{TAR}^{\mathrm{U} 23 \mathrm{C}^{\prime} \text {-endo }}$ and $\mathrm{TAR}^{\mathrm{U} 25 \mathrm{C}^{\prime} \text {-endo }}$ simulations were generated by superposition of a $\mathrm{C} 2^{\prime}$-endo uridine nucleotide onto U23/U25 in 1ANR using the uridine base atoms, and replacing the sugar-backbone atoms with those of the superimposed C2'endo uridine. For $\mathrm{TAR}^{\mathrm{U} 23 \mathrm{C}^{\prime} \text {-endo }}$ and $\mathrm{TAR}^{\mathrm{U} 25 \mathrm{C}^{\prime} \text {-endo }}$, only the backbone of the $\mathrm{C} 2^{\prime}$-endo uridine was fixed during energy minimization, while for TAR ${ }^{\text {FARFAR, }}$, all the heavy atoms were fixed during minimization.

\section{$\underline{\text { NMR residual dipolar coupling (RDC) data }}$}

The RDC data (sugar $\mathrm{C} 1^{\prime}-\mathrm{H} 1^{\prime} / \mathrm{C}^{\prime}-\mathrm{H} 2^{\prime} / \mathrm{C}^{\prime}-\mathrm{H} 33^{\prime} / \mathrm{C}^{\prime}-\mathrm{H} 4^{\prime}$ and base C8-H8/C6-H6/C2$\mathrm{H} 2 / \mathrm{C} 5-\mathrm{H} 5 / \mathrm{N} 1-\mathrm{H} 1 / \mathrm{N} 3-\mathrm{H} 3)$ used in the TAR ensemble determination were reported previously ${ }^{18,25,60}$ and are summarized in Supplementary Table 1. The raw data can also be downloaded from https://sites.duke.edu/alhashimilab/resources/. RDCs were measured using the same buffer conditions (15 mM sodium phosphate, $25 \mathrm{mM} \mathrm{NaCl}, 0.1$ $\mathrm{mM}$ EDTA, with/without $3 \mathrm{mM} \mathrm{Mg}^{2+}$ at $\mathrm{pH} \sim 6.4$ ) at $25^{\circ} \mathrm{C}$ with similar RNA concentrations 
$(\sim 1.0 \mathrm{mM})$ and slightly different $(6-22 \mathrm{mg} / \mathrm{mL})$ Pf1 phage concentrations ${ }^{18}$.

\section{$\underline{\text { RDC calculations }}$}

Ensemble-averaged RDCs were calculated by computing the RDCs for each conformer in an ensemble using the program PALES ${ }^{61}$. PALES computes RDCs based on global molecular shape. The RDC were computed using a cylindrical wall model using the following command:

$$
\begin{aligned}
& \text { pales -pdb input.pdb -ind input_rdc.tab -outd output_rdc.tab } \\
& -H-p f l-w V 0.022
\end{aligned}
$$

where $-p d b$ is the path of an input PDB file (input.pdb), $-i n D$ is the path of an input data file indicating the bond vectors for which RDC should be computed (input_rdc.tab), out $D$ is the path of the output file containing all the calculated RDCs, $-H$ means selecting all atoms including proton and $-p f 1-W V$ specifies the pfl effective concentration $(0.022 \mathrm{~g} / \mathrm{mL})$ assuming a rod liquid crystal model.

The RDC values were the averaged over all conformers in an ensemble assuming that they are equiprobable. Individual scaling factors were applied on the predicted RDCs of each construct to account for the difference of alignment magnitude in part arising due to differences in phage concentrations in the experiments, as described previously ${ }^{18}$. The elongated constructs were elongated in silico using an idealized A-form geometry prior to 
RDC calculation as described previously ${ }^{18}$.

The TAR apical loop was modeled using the wild-type CUGGGA loop ${ }^{18}$. As reported previously for the Anton-MD-NMR ensemble ${ }^{18}$, replacing the loop with the UUCG loop used to measure $\mathrm{RDCs}^{62}$ minimally impacted the RDC agreement for the FARFAR ensembles (Extended Data Fig. 1e, f). This is consistent with prior NMR studies showing that the apical loop replacement minimally impacts the dynamics of the bulge $e^{18,63}$.

\section{$\underline{\text { Sample and Select (SAS) }}$}

We used the SAS approach ${ }^{24}$ to generate ensembles from a structural pool that best satisfied the measured RDCs. Briefly, a simulated annealing Monte Carlo sampling scheme was used to select an ensemble that minimizes the cost function depicting the differences between the measured and predicted RDCs:

$$
\chi^{2}=\frac{\sum_{j} \sum_{i}^{N}\left(L_{j} \times D_{i, j}^{c a l c}-D_{i, j}^{e x p}\right)}{N}
$$

$D_{i, j}^{\text {calc }}$ and $D_{i, j}^{\text {exp }}$ are the calculated and measured RDCs, respectively, of the ith bond vector measured on the $j t h$ TAR construct, $L_{j}$ is the overall scaling factor of alignment magnitude for construct $j$, and $N$ is the total number of bond vectors. The initial effective temperature for simulation annealing was 100 and decreased by a factor of 0.9 in every step for a total of $5 \times 10^{5}$ steps. A series of SAS runs were performed varying the ensemble 
size from $N=1$ to an ensemble size in which the RDC RMSD reaches a plateau (Extended Data Fig. 1b, 2). The resultant ensemble size for different bulge variants were: $N=4$ (U1TAR, no $\left.\mathrm{Mg}^{2+}\right), N=10\left(\mathrm{U} 2-\mathrm{TAR}\right.$, no $\left.\mathrm{Mg}^{2+}\right), N=20\left(\mathrm{TAR}\right.$, no $\left.\mathrm{Mg}^{2+}\right), N=10(\mathrm{U} 7-\mathrm{TAR}$, no $\left.\mathrm{Mg}^{2+}\right), N=4\left(\mathrm{U} 1-\mathrm{TAR}\right.$, with $\left.\mathrm{Mg}^{2+}\right), N=5\left(\mathrm{TAR}\right.$, with $\mathrm{Mg}^{2+}$ ) and $N=5$ (U7-TAR, with $\mathrm{Mg}^{2+}$ ). To analyze distributions of structural parameters (Fig. 3 and Extended Data Fig. $3,4)$, we also generated larger sized ensembles by running SAS multiple times to ensure the total size $N=2,000$ for all systems.

The SAS analysis excluded RDCs from the flexible terminal G17-C45 bp as well as those of the C29-G36 bp flanking the apical loop, given differences between the apical loop sequences used to measure RDCs (wild-type or UUCG) and to model (wild-type) TAR. Note that RDCs from G17-C45 and C29-G36 bp were included in the prior study ${ }^{18}$ and this explains the small differences in RDC RMSD for the two Anton-MD derived ensembles relative to that reported earlier.

\section{$\underline{\text { Cross-validation analysis }}$}

Cross-validation was performed using two approaches as described previously ${ }^{18}$. In one approach (inactive random, Extended Data Fig. 1c, d), 10\% of the RDC data was randomly removed and SAS was used to generate an ensemble. The RMSD between measured and predicted RDCs was then computed for the left out RDC data. This procedure was repeated 10 times and the final RDC RMSD was averaged over all 10 independent runs. For TAR (absence of $\mathrm{Mg}^{2+}$ ) where we have RDCs measured on four 
constructs, a second mode (inactive media, Extended Data Fig. 1c, d) of cross-validation was also performed, in which the RDC dataset of each construct was left out individually before running SAS. The RMSD between measured and predicted RDCs was then computed for the left out RDC dataset. The final RDC RMSD is averaged over the four iterations corresponding to leaving out each RDC dataset.

\section{$\underline{\text { NMR chemical shift data }}$}

The ${ }^{1} \mathrm{H},{ }^{13} \mathrm{C}$ and ${ }^{15} \mathrm{~N}$ chemical shift assignments of TAR have been published previously ${ }^{40,63}$ and were compared to quantum mechanical chemical shift predictions. The numerical populations for $\mathrm{C}^{2}$-endo shown in Fig. 3e were obtained based on the C1' chemical shift assuming a linear dependence between the range $86 \mathrm{ppm}\left(100 \% \mathrm{C} 2^{\prime}\right.$-endo $)$ 48 and 94 ppm $\left(100 \% \mathrm{C}^{\prime} \text {-endo }\right)^{40}$.

Automated fragmentation quantum mechanics/ molecular mechanics (AF-QM/MM) $\underline{\text { chemical shift calculation }}$

Chemical shift calculations were performed using a previously described fragmentation procedure ${ }^{37}$. Each RNA structure was subjected to 5 steps of conjugate gradient minimization with harmonic restraints of $2 \mathrm{kcal} / \mathrm{mol}-\AA^{2}$ on all heavy atoms; this regularizes bond lengths and angles to minimize noise in the results that can arise from very small changes in these geometric parameters. Next, each structure was broken into "quantum" fragments centered on each nucleotide, containing 2-6 neighboring nucleotides, 
using a heavy-atom distance cutoff of $3.4 \AA$. The effects of RNA atoms outside the quantum region, and of water and ions in the solvent, were represented as point charges uniformly distributed on the molecular surface of the quantum region and resolved by fitting to Poisson-Boltzmann calculations using the "solinprot" program from the MEAD package ${ }^{64,65}$. The quantum region was assigned a local dielectric $\varepsilon$ of 1 (vacuum); the remaining RNA region had an $\varepsilon$ of 4 , and the solvent region an $\varepsilon$ of 80 . GIAO chemical shift calculations were carried out for each fragment, using version 5.0 of the demon- $2 \mathrm{k}$ program $^{66}$ using the OLYP functional ${ }^{67}$ with the pcSseg-1 (triple- $\varsigma$ plus polarization) basis set optimized for chemical shifts ${ }^{68}$ for the central nucleotide (whose results are reported here), and a DZVP basis for the remaining atoms. Reference shieldings were computed for tetramethylsilane (TMS) using the same functional and basis set. The ensembles of Anton-MD-NMR, FARFAR-NMR as well as a randomly selected Anton-MD and FARFAR-library of size $N=20$ were examined. Note that $\mathrm{R}^{2}$ can be artificially low for spins with small ranges of chemical shifts (e.g. $\sim 1 \mathrm{ppm}$ for $\mathrm{C}^{\prime} / \mathrm{C} 4^{\prime} / \mathrm{C} 6$ for central WatsonCrick bps).

\section{$\underline{\text { Ensemble analysis }}$}

The local backbone and sugar torsion angles (Fig. 3b) were calculated using X3DNA$\operatorname{DSSR}^{69}$. The inter-helical Euler angles $\left(\alpha_{\mathrm{h}}, \beta_{\mathrm{h}}, \gamma_{\mathrm{h}}\right)$ were computed as described previously ${ }^{35}$ (Fig. 1g, Fig. 4b, c, Extended Data Fig. 3, 4, 7). Briefly, the upper helix from G26 to G28 and lower helix from C19 to G21 are aligned to idealized A-form helix, 
respectively. The sign of $\alpha_{\mathrm{h}}$ and $\gamma_{\mathrm{h}}$ is inverted relative to previously reported values ${ }^{18}$ such that a positive and negative inter-helical twist angle $\left(\alpha_{h}+\gamma_{h}\right)$ corresponds to over- and under-twisting, respectively ${ }^{70}$.

Junctional topology (Extended Data Fig. 7) was defined as the base pairing mode which is detected by X3DNA-DSSR ${ }^{69}$. If two bases are forming a base pair with LeontisWesthof (LW) classification as "cWW" (e.g. Watson-Crick base pair, Wobble base pair), a solid line is indicated between the two bases (Extended Data Fig. 7), whereas other LW classifications are indicated as a dash line (Extended Data Fig. 7).

TAR conformers were considered to be co-axially stacked when the bases comprising A22-U40 and G26-C39 or U25-U40, were stacked with each other, as defined by X3DNA$\operatorname{DSSR}^{69}$.

The lower bound estimate of pairwise RMSD between two ensembles was defined as the following: Consider two ensembles $\mathrm{A}$ and $\mathrm{B}$ with size $N_{\mathrm{A}}$ and $N_{\mathrm{B}}$. For every conformer in ensemble A, we found the corresponding conformer in ensemble B that has the lowest pairwise RMSD. This procedure was repeated for every conformer in A to obtain a total of $N_{\mathrm{A}} \mathrm{RMSD}$ values. We then took the root mean square of all these $N_{\mathrm{A}}$ RMSD values. The procedure was also repeated considering ensemble B. Then the minimum of the two root mean square values was selected as a lower bound of the similarity between the two ensembles. By this definition, Anton-MD-NMR and FARFAR-NMR ensembles differ by $3.7 \AA$ while ensembles obtained from multiple independent FARFAR-NMR runs typically differ by $1.4 \AA$ on average. 
The cooperativity of flipping bulge nucleotides out was computed from the FARFAR ensemble as follows - For U23, C24 and U25, the probability of independently flipping out was computed as:

$$
P(\text { nt out })=\frac{N(\text { nt out } \mid \text { other } n t \text { in })}{N(\text { nt out } \mid \text { other } n t \text { in })+N(n t \text { in } \mid \text { other } n t \text { in })}
$$

where $N(n t$ out $\mid$ other $n t$ in) is the number of FARFAR-NMR conformers with the nucleotide (nt) of interest being flipped out with the other nucleotides (among U23, C24 and U25) being flipped in and $N(n t$ in $\mid$ other $n t$ in) is the number of FARFAR-NMR conformers with U23, C24 and U25 flipped in. Flipping in and flipping out of U23, C24 and U25 were gauged by visual examination of the conformers (Extended Data Fig. 7). The probability of simultaneously flipping out U23, C24 and U25 without cooperativity as then computed as the product of the probabilities of independently flipping out each nucleotide as defined above i.e.,

$$
P(U 23, C 24, U 25 \text { out })=P(U 23 \text { out }) * P(C 24 \text { out }) * P(U 25 \text { out })
$$

This was then compared to the observed probability of U23, C24 and U25 being flipped out $P(U 23, C 24, U 25$ out obs $)$ which was computed as the fraction of FARFARNMR conformers with U23, C24 and U25 flipped out (8/20). Cooperativity was defined as: 


$$
-R T \ln \left(\frac{P(U 23, C 23, U 25 \text { out obs })}{P(U 23, C 23, U 25 \text { out })}\right)
$$

where $R$ is the universal gas constant, $T$ is temperature in Kelvin $(298 \mathrm{~K})$.

\section{$\underline{\text { NMR sample preparation }}$}

TAR, TAR-Nm-U23, and TAR-Nm-C24 RNA samples were synthesized using a MerMade 6 Oligo Synthesizer (BioAutomation) via solid-phase synthesis using standard phosphoramidite chemistry and deprotection protocols ${ }^{71}$. 2'-TBDMS protected phosphoramidites (ChemGenes) and $1 \mu \mathrm{mol}$ standard synthesis columns (1000 $\AA$ ) were used. The final 5'-DMT (4,4'-dimethoxytrityl) was removed during the synthesis for DMT-off deprotection and PAGE purification. Removal of nucleobase and phosphate protecting groups, and cleavage from the $1 \mu \mathrm{mol}$ columns was achieved using $1 \mathrm{ml}$ of $30 \%$ ammonium hydroxide and 30\% methylamine (1:1) followed by 2 -hour incubation at room temperature. The solution was then air-dried and dissolved in $100 \mu \mathrm{L}$ DMSO and $125 \mu \mathrm{L}$ TEA-3HF, followed by $2.5 \mathrm{~h}$ incubation at $65{ }^{\circ} \mathrm{C}$ following Glen Research protocols (https://www.glenresearch.com/reports/gr19-22) for 2'-O deprotection. The sample was then ethanol precipitated overnight, air dried, then dissolved in water for gel purification using a $20 \%(\mathrm{w} / \mathrm{v})$ polyacrylamide gel with $8 \mathrm{M}$ urea and $1 \mathrm{x}$ Tris/borate/EDTA. The RNA was removed from the excised gel by electro-elution in $1 \mathrm{x}$ Tris/acetic acid/EDTA followed by ethanol precipitation. The RNA was annealed in water at a concentration of 
$50 \mu \mathrm{M}$ by heating at $95^{\circ} \mathrm{C}$ for $5 \mathrm{~min}$ followed by cooling on ice for $60 \mathrm{~min}$. It was then buffer exchanged using an Amicon Ultra-15 centrifugal filter (EMD Milipore) with a 3 $\mathrm{kDa}$ cutoff into NMR buffer (15mM sodium phosphate, $25 \mathrm{mM} \mathrm{NaCl}, 0.1 \mathrm{mM}$ EDTA) at $\mathrm{pH}$ 6.4. The final concentrations were: $\sim 0.8 \mathrm{mM}$ for TAR, $2.5 \mathrm{mM}$ TAR-Nm-U23 and 1.4 mM TAR-Nm-C24. TAR was also buffer exchanged into NMR buffer containing 3 $\mathrm{mM} \mathrm{Mg}{ }^{2+}$.

\section{$\underline{\text { NMR spectroscopy }}$}

All the NMR 2D HSQC experiments in this study were carried out on Bruker Avance III 600-MHz NMR spectrometer equipped with a triple-resonance cryogenic probed at $25^{\circ} \mathrm{C}$. NMR Data were processed using NMRpipe ${ }^{72}$ and analyzed using SPARKY (T.D. Goddard and D.G. Kneller, SPARKY 3, University of California, San Francisco), respectively. The resonance assignments for Nm-modified TAR were obtained based on the previous reported assignments of $\mathrm{TAR}^{63}$ and further confirmed using 2D NOESY experiments. 


\section{Methods references:}

Salomon-Ferrer, R., Gotz, A.W., Poole, D., Le Grand, S., \& Walker, R.C., Routine Microsecond Molecular Dynamics Simulations with AMBER on GPUs. 2. Explicit Solvent Particle Mesh Ewald. J. Chem. Theory Comput. 9 (9), 3878-3888 (2013). Jorgensen, W.L., Chandrasekhar, J., Madura, J.D., Impey, R.W., \& Klein, M.L., Comparison of Simple Potential Functions for Simulating Liquid Water. J. Chem. Phys. 79 (2), 926-935 (1983).

Joung, I.S. \& Cheatham, T.E., 3rd, Determination of alkali and halide monovalent ion parameters for use in explicitly solvated biomolecular simulations. J. Phys. Chem. B 112 (30), 9020-9041 (2008).

Rangadurai, A. et al., Why are Hoogsteen base pairs energetically disfavored in ARNA compared to B-DNA? Nucleic Acids Res. 46 (20), 11099-11114 (2018).

Abraham, M.J. et al., GROMACS: High performance molecular simulations through multi-level parallelism from laptops to supercomputers. SoftwareX 1-2, 1925 (2015).

Berendsen, H.J.C., van der Spoel, D., \& van Drunen, R., GROMACS: A messagepassing parallel molecular dynamics implementation. Comput. Phys. Commun. 91 (1), 43-56 (1995).

Piana, S., Donchev, A.G., Robustelli, P., \& Shaw, D.E., Water Dispersion Interactions Strongly Influence Simulated Structural Properties of Disordered Protein States. J. Phys. Chem. B 119 (16), 5113-5123 (2015).

MacKerell, A.D.J. et al., All-Atom Empirical Potential for Molecular Modeling and Dynamics Studies of Proteins. J. Phys. Chem. B 102 (18), 3586 (1998).

Berendsen, H.J.C., Postma, J.P.M., van Gunsteren, W.F., DiNola, A., \& Haak, J.R., Molecular dynamics with coupling to an external bath. J. Chem. Phys. 81, 36843690 (1984).

Bussi, G., Donadio, D., \& Parrinello, M., Canonical sampling through velocity rescaling. J. Chem. Phys. 126 (1) (2007).

Nose, S. \& Klein, M.L., Constant Pressure Molecular-Dynamics for MolecularSystems. Mol. Phys. 50 (5), 1055-1076 (1983).

Parrinello, M. \& Rahman, A., Polymorphic Transitions in Single-Crystals - a New Molecular-Dynamics Method. J. Appl. Phys. 52 (12), $7182-7190$ (1981).

Darden, T., York, D.M., \& Pedersen, L., Particle mesh Ewald: An N·log(N) method for Ewald sums in large systems. J. Chem. Phys. 103, 10089-10092 (1993).

Hess, B., Bekker, H., Berendsen, H.J.C., \& Fraaije, J.G.E.M., LINCS: A linear constraint solver for molecular simulations. J. Comput. Chem. 18 (12), 1463-1472 (1997).

Roe, D.R. \& Cheatham, T.E., PTRAJ and CPPTRAJ: Software for Processing and Analysis of Molecular Dynamics Trajectory Data. J. Chem. Theory Comput. 9 (7), 3084-3095 (2013). 
60 Dethoff, E.A., Hansen, A.L., Zhang, Q., \& Al-Hashimi, H.M., Variable helix elongation as a tool to modulate RNA alignment and motional couplings. J. Magn. Reson. 202 (1), 117-121 (2010).

61 Zweckstetter, M. \& Bax, A., Predicition of sterically induced alignment in a dilute liquid crystalline phase; aid to protein structure determination by NMR. J. Am. Chem. Soc. 122, 3791-3792 (2000).

62 Salmon, L. et al., Modulating RNA Alignment Using Directional Dynamic Kinks: Application in Determining an Atomic-Resolution Ensemble for a Hairpin using NMR Residual Dipolar Couplings. J. Am. Chem. Soc. (2015).

63 Dethoff, E.A. et al., Characterizing complex dynamics in the transactivation response element apical loop and motional correlations with the bulge by NMR, molecular dynamics, and mutagenesis. Biophys. J. 95 (8), 3906-3915 (2008).

64 Bashford, D. \& Karplus, M., pKa's of ionizable groups in proteins: atomic detail from a continuum electrostatic model. Biochemistry 29 (44), 10219-10225 (1990).

65 Richardson, W.H., Peng, C., Bashford, D., Noodleman, L., \& Case, D.A., Incorporating solvation effects into density functional theory: Calculation of absolute acidities. Int. J. Quantum Chem. 61, 207-217 (1998).

66 Koster, A.M., Geudtner, G., Calaminici, P., Casida, M. E., Dominguez, V. D., Flores-Moreno, R., Gamboa, G. U., Goursot, A. H. T., Ipatov, A. J. F., del Campo, J. M., Reveles, J. U., Vela, A., Zuniga-Gutierrez, B., and Salahub, D. R., deMon2k, version 5. (Cinvestav, Mexico City, 2011).

67 Handy, N.C. \& Cohen, A.J., Left-right correlation energy. Mol. Phys. 99 (5), 403412 (2001).

68 Jensen, F., Segmented Contracted Basis Sets Optimized for Nuclear Magnetic Shielding. J. Chem. Theory Comput. 11 (1), 132-138 (2015).

69 Lu, X.J., Bussemaker, H.J., \& Olson, W.K., DSSR: an integrated software tool for dissecting the spatial structure of RNA. Nucleic Acids Res. 43 (21) (2015).

70 Zhou, H. et al., New insights into Hoogsteen base pairs in DNA duplexes from a structure-based survey. Nucleic Acids Res. 43 (7), 3420-3433 (2015).

71 Damha, M.J. \& Ogilvie, K.K., Oligoribonucleotide synthesis. The silylphosphoramidite method. Methods Mol. Biol. 20, 81-114 (1993).

72 Delaglio, F. et al., Nmrpipe - a Multidimensional Spectral Processing System Based On Unix Pipes. J. Biomol. NMR 6 (3), 277-293 (1995).

73 Al-Hashimi, H.M. et al., Concerted motions in HIV-1 TAR RNA may allow access to bound state conformations: RNA dynamics from NMR residual dipolar couplings. J. Mol. Biol. 315 (2), 95-102. (2002). 
Acknowledgments: We thank members of the Al-Hashimi laboratory and Dr. Dawn Merriman (University of Dayton) for assistance and critical comments on the manuscript. We would like to thank Dr. Andrew Watkins (Stanford University) for advice about FARFAR. Funding: This work was supported by US National Institute for General Medical Sciences (1R01GM132899 to H.M.A. and D.H.), and US National Institute of Health (U54 AI150470 to H.M.A. and D.A.C.) and by Tobacco Settlement Fund (21-57340010 to J.D.Y.).

Author contributions: H.S., D.H., J.D.Y. and H.M.A. conceived the project and experimental design. H.S. and J.D.Y. performed FARFAR calculations. A.R. performed MD simulations. H.S. performed sample and select and other ensemble analysis, with assistance from R.R. H.A.A. prepared NMR samples, performed NMR experiments and analyzed NMR data. D.A.C. performed QM/MM chemical shift calculation. H.M.A. and H.S. wrote the manuscript with critical input from A.R., D.H., D.A.C. and J.D.Y.

Competing interests: H.M.A. is advisor to and holds an ownership interest in Nymirum, an RNA-based drug discovery company. The remaining authors declare no competing interests. 
Materials \& Correspondence: Rosetta FARFAR commands as well as its required input files are included in Supplementary Materials. The raw RDC data can be downloaded from https://sites.duke.edu/alhashimilab/resources/. The AFNMR programs are available at https://github.com/dacase/afnmr. All other in-house Python scripts for SAS, structural analysis as well as other data analysis are available on request to the corresponding authors. The authors declare that the data supporting the findings are available within the article and its Supplementary Materials, or are available upon request to the corresponding authors. 


\section{Extended Data Figures}
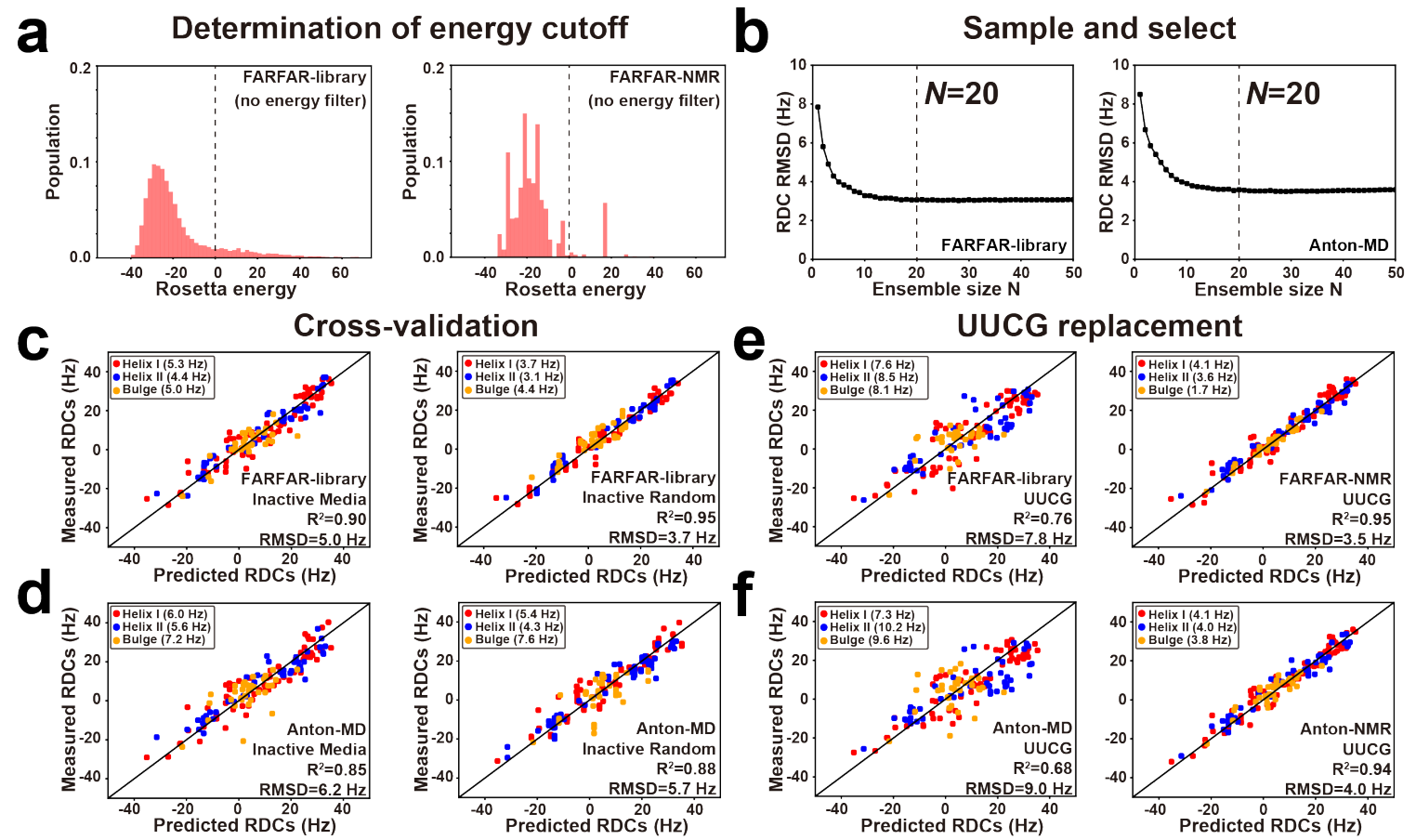

Extended Data Fig. 1 Optimizing TAR ensembles using SAS. (a) Determination of

Rosetta energy cutoff. (left) Shown are the distribution of energies of a FARFAR-library (no energy filter) $(N=10,000)$ generated without pre-filtering based on Rosetta energy (i.e., not excluding structures with Rosetta energy $>0$ ). (right) Distribution of no pre-filtering FARFAR-library in (left) after RDC selection $(N=2,000)$. The Rosetta energy $=0$ is indicated using a dashed line. As structures with energy $>0$ were predominantly excluded following SAS, only structures with energy $<0$ were retained while generating the FARFAR-library in the main manuscript. (b) RDC RMSD as a function of ensemble size $(N)$ during SAS for (left) the FARFAR-library and (right) Anton-MD. The chosen ensemble size $N=20$ is indicated using a vertical dashed line. (c-d) Cross-validation analysis of TAR ensembles. Shown are comparison of measured and predicted RDCs obtained from cross-validation analysis on TAR ensembles using two modes: Inactive Media (left) and Inactive Random (right)(Methods) for (c) the FARFAR-library and (d) Anton-MD. (e-f) Generation of TAR ensembles using UUCG apical loop models. 
Comparison between measured and predicted RDCs for starting pools with $N=10,000$ (left) and comparison between measured and predicted RDCs after SAS with $N=20$ (right) from (e) FARFAR-library replacing wild-type CUGGGA loop with a UUCG loop ${ }^{62}$ and (f) Anton-MD replacing wild-type CUGGGA loop with a UUCG loop. Replacing the loop with the UUCG loop used to measure RDCs minimally impacted the RDC agreement for both FARFAR and Anton ensembles. 


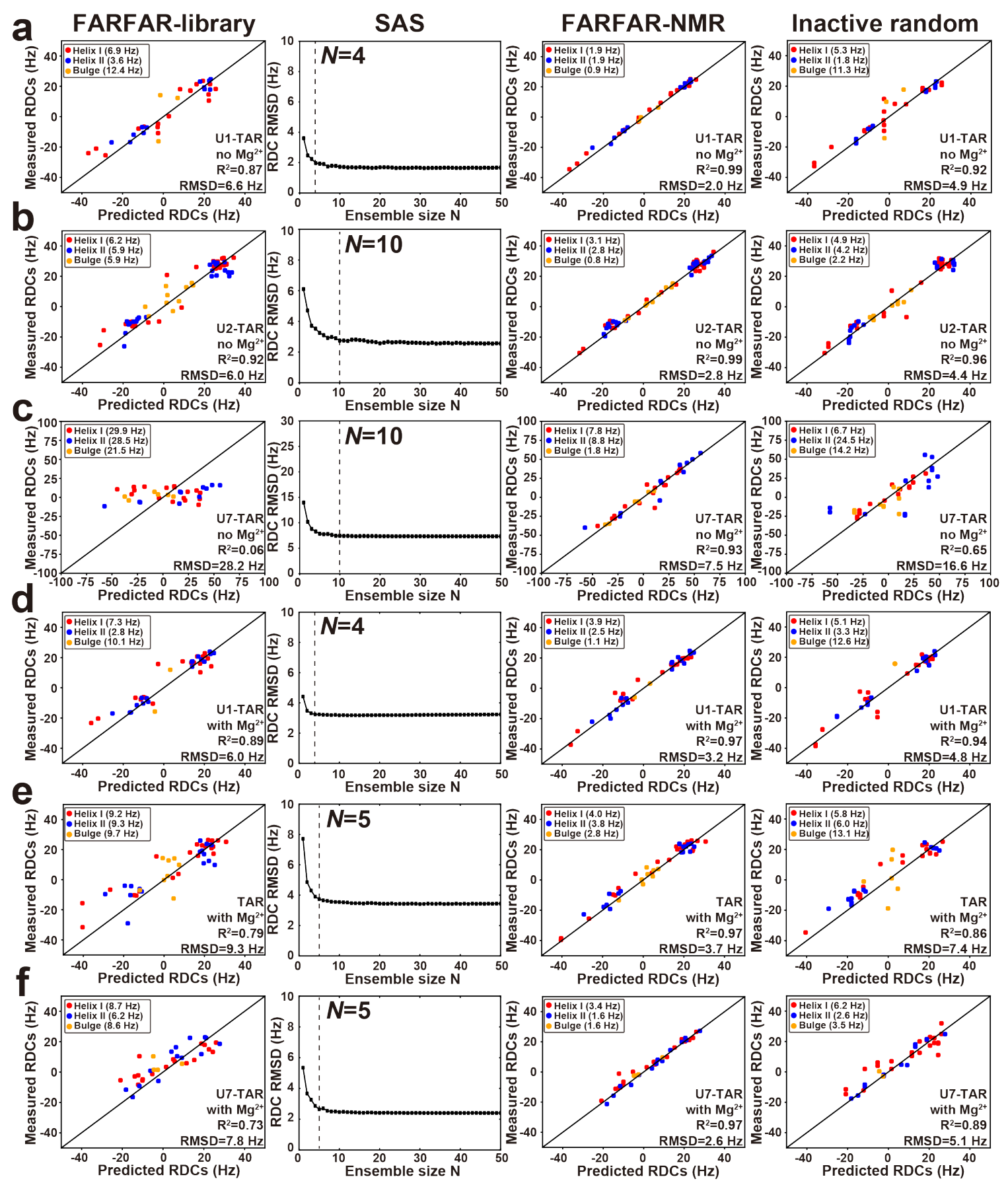

Extended Data Fig. 2 Optimizing ensembles of TAR and its variants. From left to right, comparison of measured and predicted RDCs for the FARFAR-library $(N=10,000)$, RDC RMSD as a function of ensemble size during SAS (ensemble size $N$ chosen for the final ensembles is indicated as a vertical dashed line), comparison between the measured and predicted RDCs for the FARFAR-NMR ensembles after SAS, and inactive random 
cross-validation for (a) U1-TAR no $\mathrm{Mg}^{2+}$ (b) U2-TAR no $\mathrm{Mg}^{2+}$ (c) U7-TAR no $\mathrm{Mg}^{2+}$ (d) U1-TAR with $\mathrm{Mg}^{2+}$ (e) TAR with $\mathrm{Mg}^{2+}$ and (f) U7-TAR with $\mathrm{Mg}^{2+}$. RDC values are color coded according to structural element as defined in Fig. 1a and Extended Data Figure $3 a$. 

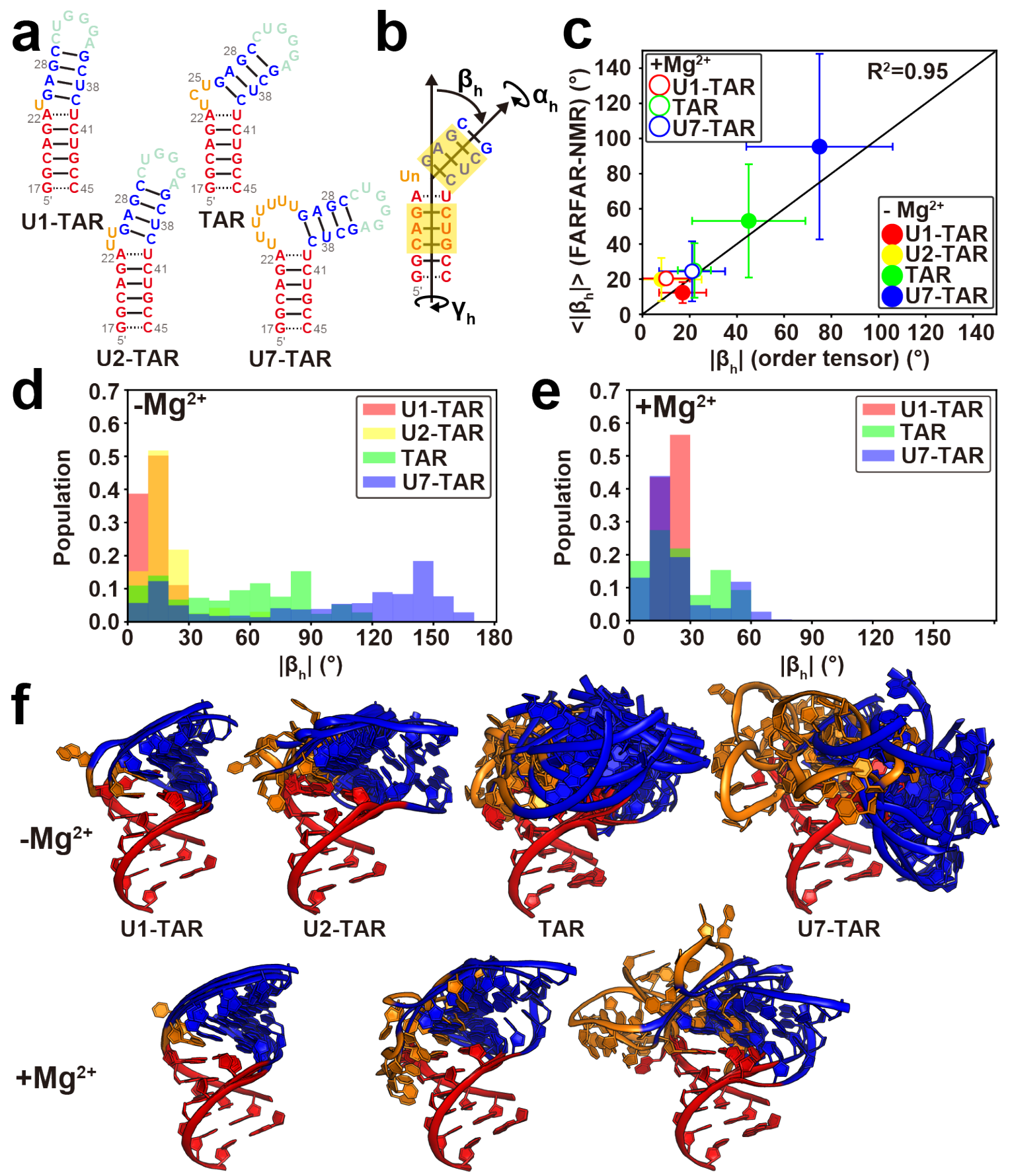

U1-TAR

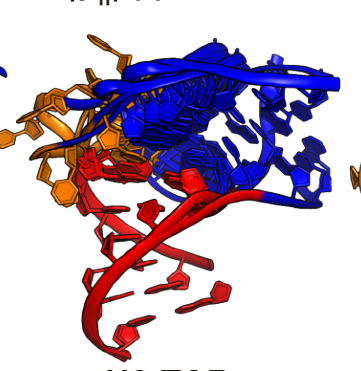

U2-TAR

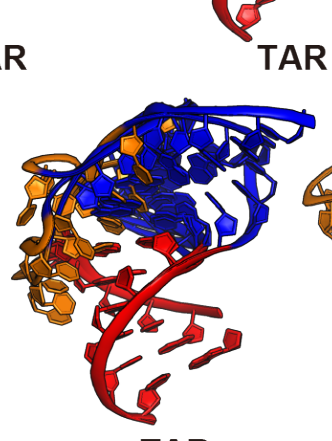

TAR 
Comparison between the average bend angle $\left(<\left|\beta_{\mathrm{h}}\right|>\right)$ and its standard deviation for the FARFAR-NMR $(N=2,000)$ derived ensembles with values obtained from an order tensor analysis of the $\mathrm{RDCs}^{28}$. The standard deviation in the order tensor analysis corresponds to half the cone radius angle assuming an isotropic model ${ }^{73}$. (d-e) Distributions of the inter-helical bend angle magnitude $\left|\beta_{\mathrm{h}}\right|$ for each ensemble $(N=2,000)$ in the absence (d) and presence (e) of $\mathrm{Mg}^{2+}$. (f) Ensembles of TAR and its bulge variants in the absence (upper) and presence (lower) of $3 \mathrm{mM} \mathrm{Mg}^{2+}$. Motifs are color-coded as in (a). 

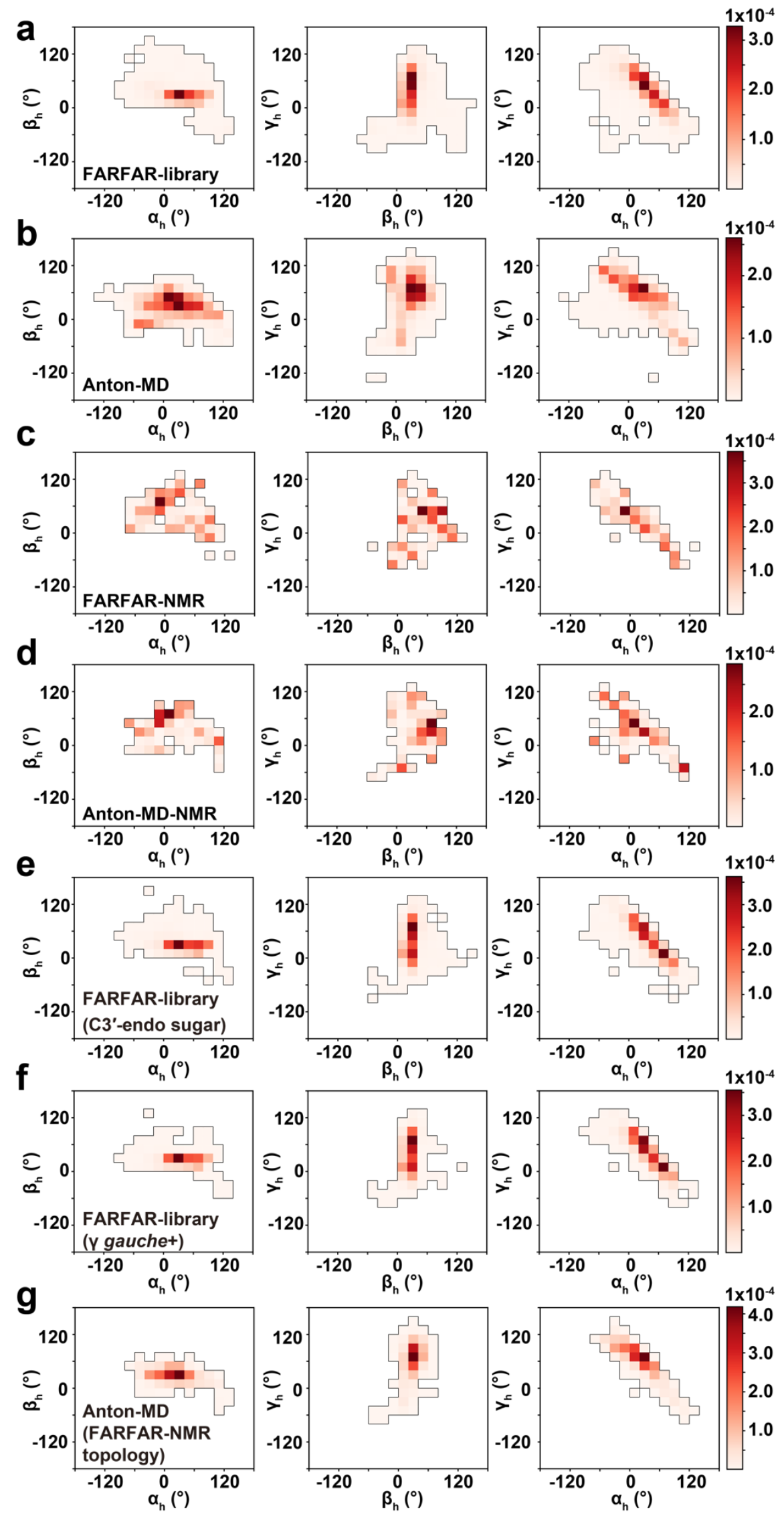


\section{Extended Data Fig. 4 Inter-helical orientational distributions of FARFAR and Anton}

libraries and ensembles. The $2 \mathrm{D}$ density map of inter-helical Euler angle $\left(\alpha_{\mathrm{h}}, \beta_{\mathrm{h}}, \gamma_{\mathrm{h}}\right)$ for (a) FARFAR-library $(N=10,000)$, (b) Anton-MD $(N=10,000)$, (c) FARFAR-NMR $(N=$ 2,000), (d) Anton-MD-NMR ( $N=2,000)$, (e-f) two subsets of FARFAR-library: (e) one with U23, C24, U25, A22 and U40 constrained to be C3'-endo $(N=4,422)$, and (f) the other one with U23, C24, U25, A22 and U40 constrained to be only gauche $+\gamma\left(20^{\circ}<\gamma<100^{\circ}\right)$ $(N=3,145)$, (g) a subset of Anton-MD with conformers retaining the same junction topology as that in FARFAR-NMR (Methods) $(N=2,074)$. The color-scale for density is given on the right. In all cases, the bin width is $20^{\circ}$. 

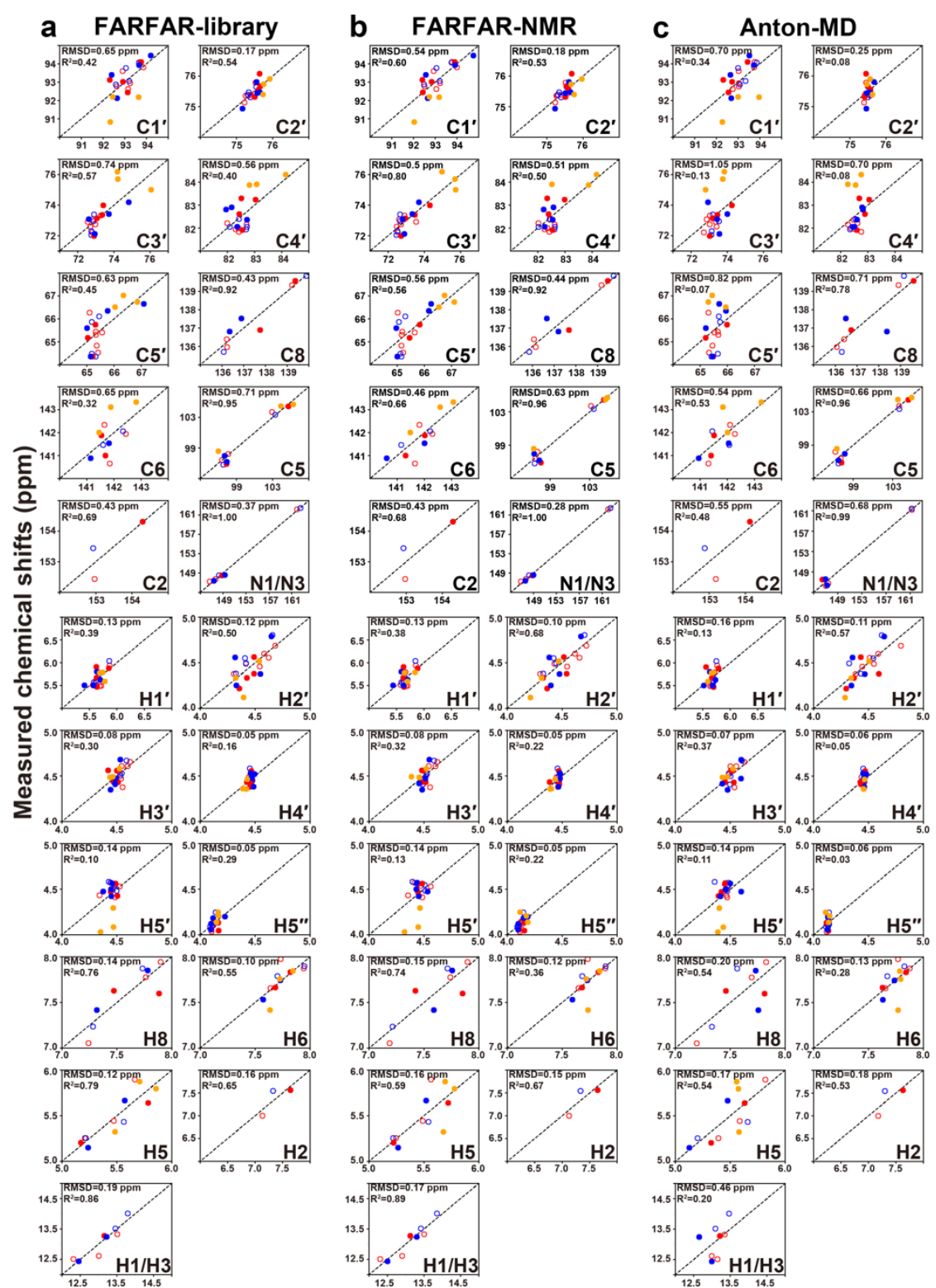

\section{d Anton-MD-NMR}
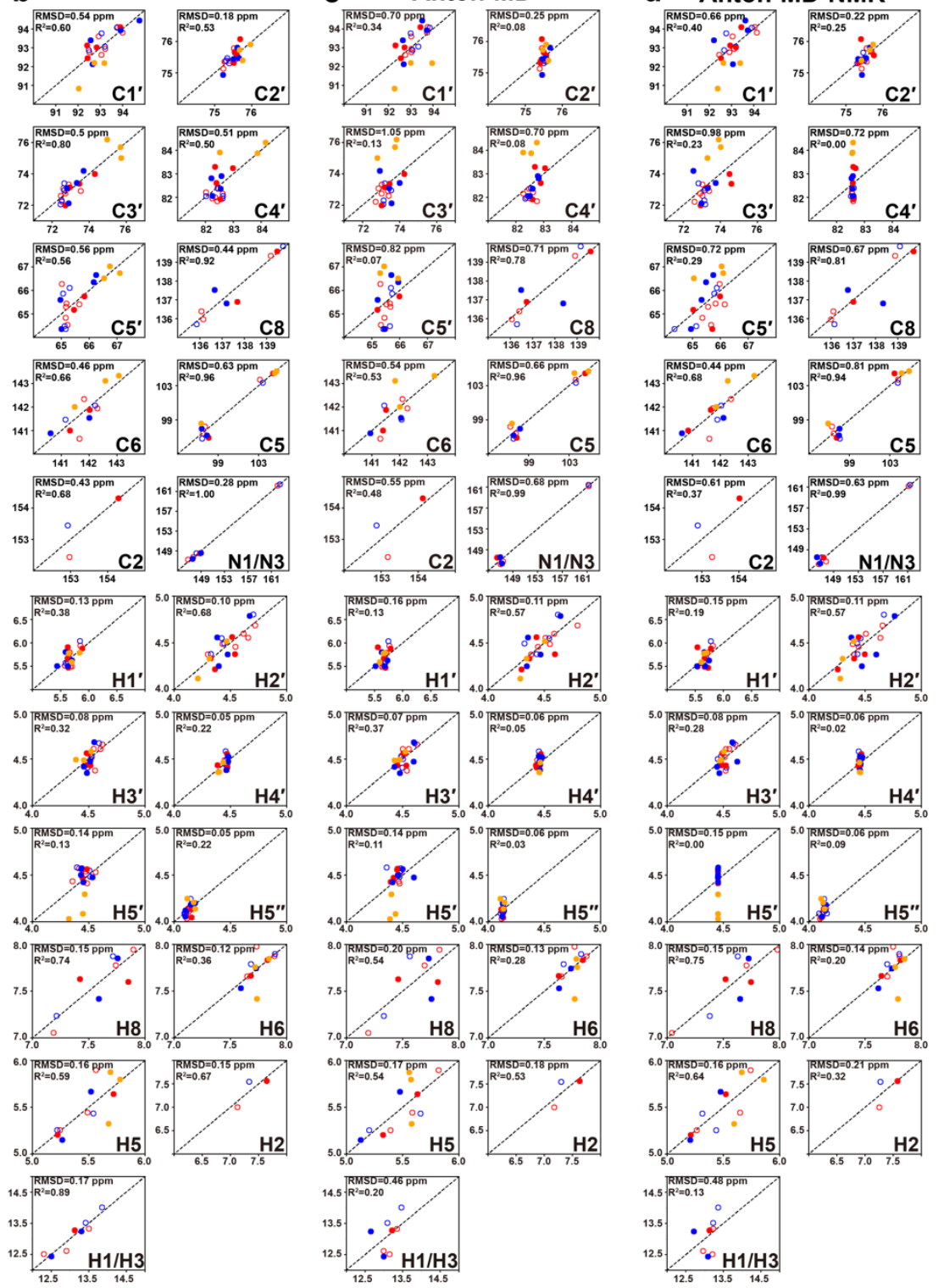

Predicted chemical shifts (ppm)

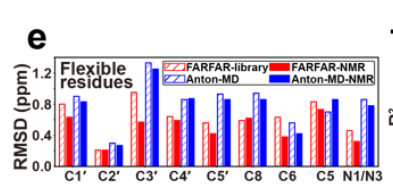

f Predicted chemicas

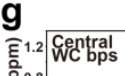

h
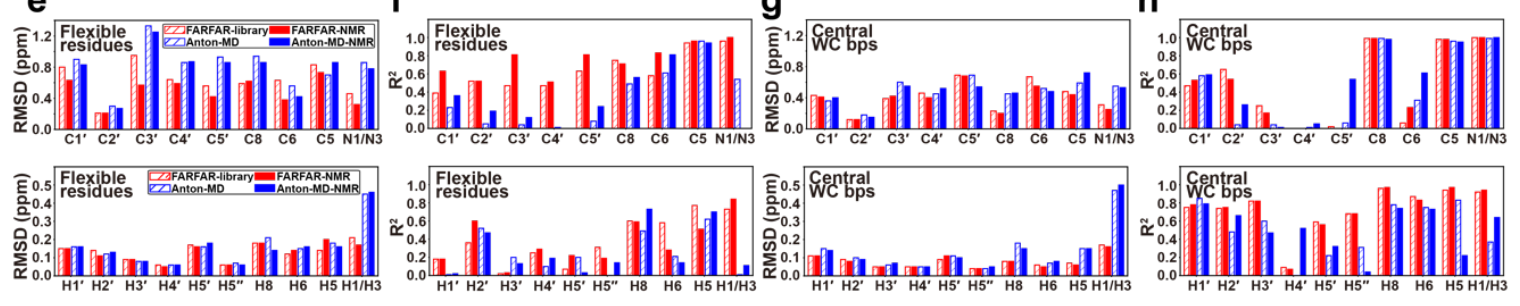

\section{Extended Data Fig. 5 Evaluation of TAR libraries and ensembles via ${ }^{13} \mathrm{C},{ }^{15} \mathrm{~N}$ and ${ }^{1} \mathrm{H}$}

chemical shifts. (a-d) Comparison of measured and predicted ${ }^{13} \mathrm{C},{ }^{15} \mathrm{~N}$ and ${ }^{1} \mathrm{H}$ chemical shifts for (a) FARFAR-library, (b) the FARFAR-NMR ensemble, (c) Anton-MD, and (d) 
the Anton-MD-NMR ensemble ( $N=20$ in all cases) by AF-QM/MM (Methods). Chemical shifts are color-coded according to the different structural elements (Fig. 1a). Chemical shifts for the central Watson-Crick bps within A-form helices (C19-G43, A20U42, G21-C41, A27-U38, G28-C37) are denoted using open circles. A correction was applied to the predicted chemical shifts (Methods) as described previously ${ }^{38}$. (e-h) Comparison of RMSD and $\mathrm{R}^{2}$ between measured and predicted ${ }^{13} \mathrm{C} /{ }^{15} \mathrm{~N}$ (top) and ${ }^{1} \mathrm{H}$ (below) chemical shifts for (e-f) flexible residues (U23, C24, U25, A22-U40, G26-C39, C29-G36, G18-C44) and (g-h) central Watson-Crick bps for the FARFAR-library (red, open), FARFAR-NMR ensemble $(N=20)$ (red, fill), Anton-MD (blue, open) and the Anton-MDNMR ensemble $(N=20)$ (blue, fill). 


\section{Flexible residues}

a

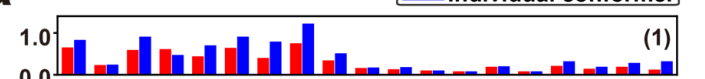

1.0 (2)

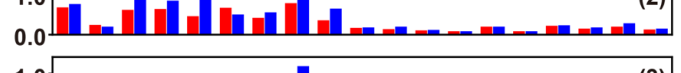

1.0
0.0
1.0

1.0 (4)

0.0 m nom

1.0 (5)

$1 . 0 \longdiv { 1 6 \text { (6) } }$

1.0

0.0

$1 . 0 \longdiv { 1 }$

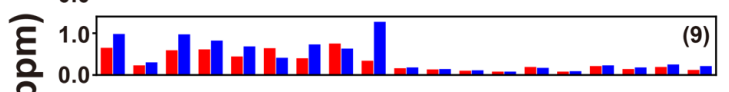

o 0.0 (10)

$\sum^{\infty} 1.0$ (11)

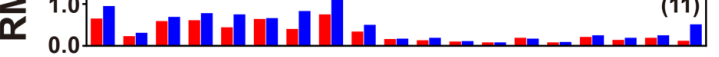

1.0 (12)

$1 . 0 \longdiv { 1 1 3 | }$

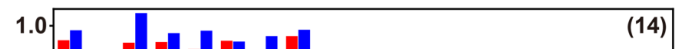

0.0

1.0 (15)

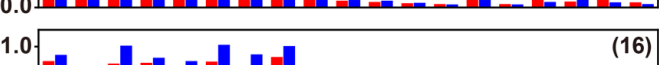

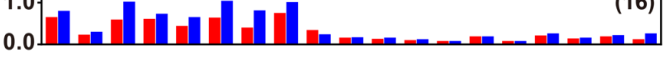

1.00 (17)

1.0 (18)

0.0

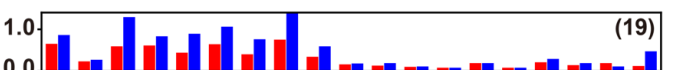

1.00 (20)

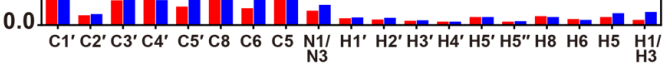

b

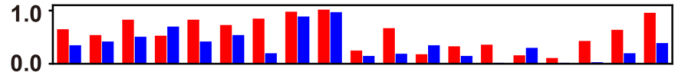

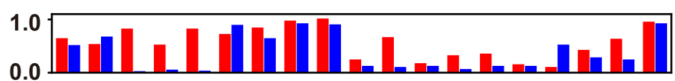

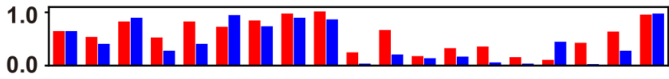

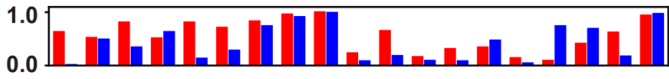

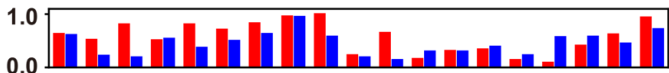

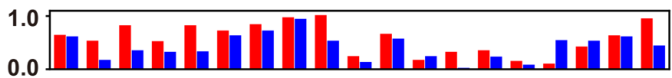

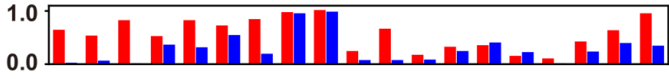

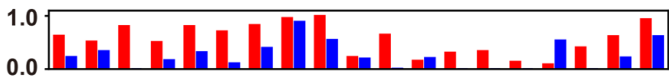

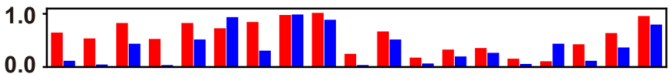

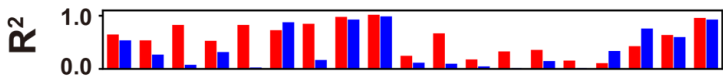

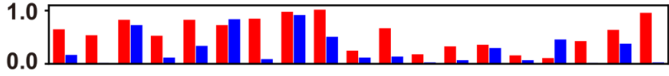
0.0 1. 1.0

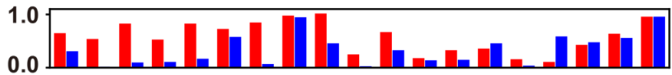
0.0 1.

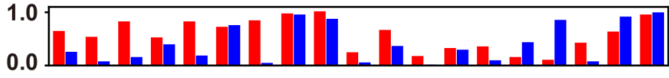

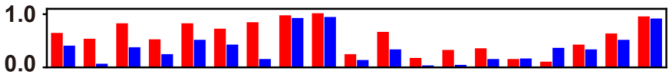

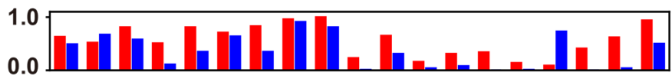

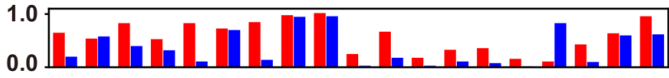

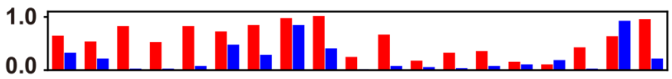
$0.0 \frac{1.0}{C 1^{\prime} C 2^{\prime} C 3^{\prime} C 4^{\prime} C 5^{\prime} C 8} C_{C 6}$

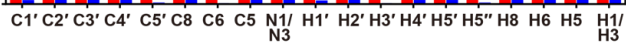

Extended Data Fig. 6 Comparison of agreement of measured and predicted chemical shifts between FARFAR-NMR ensemble and individual conformers. Bar plot of (a) RMSD and (b) $\mathrm{R}^{2}$ between measured and predicted chemical shifts for FARFAR-NMR ensemble (red) and its individual conformer (blue) for only flexible residues (U23, C24, 
bioRxiv preprint doi: https://doi.org/10.1101/2020.05.13.092981; this version posted May 23, 2020. The copyright holder for this preprint (which was not certified by peer review) is the author/funder. All rights reserved. No reuse allowed without permission.

U25, A22-U40, G26-C39, C29-G36, G18-C44). All the conformers in FARFAR-NMR ensemble $(N=20)$ are sorted in increasing order of the bend angle magnitude $\left|\beta_{\mathrm{h}}\right|$. 
a FARFAR-NMR

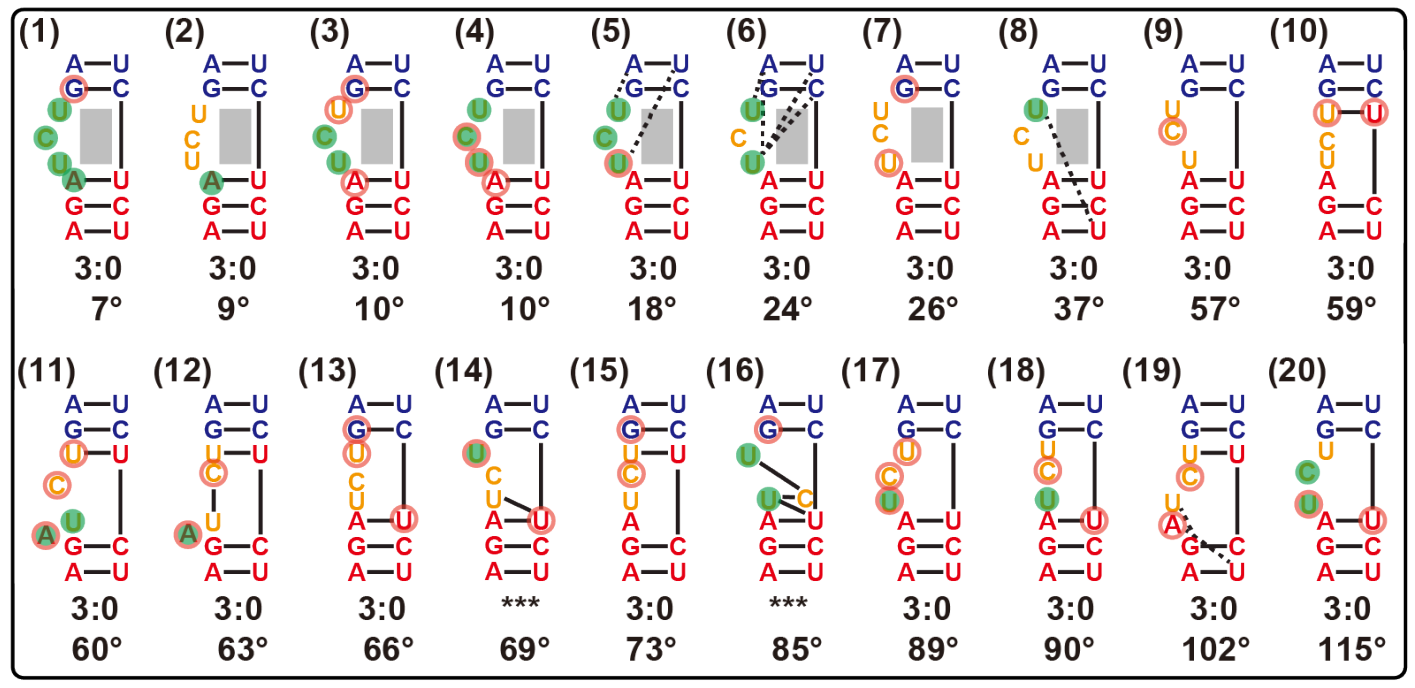

\section{b Anton-MD-NMR}

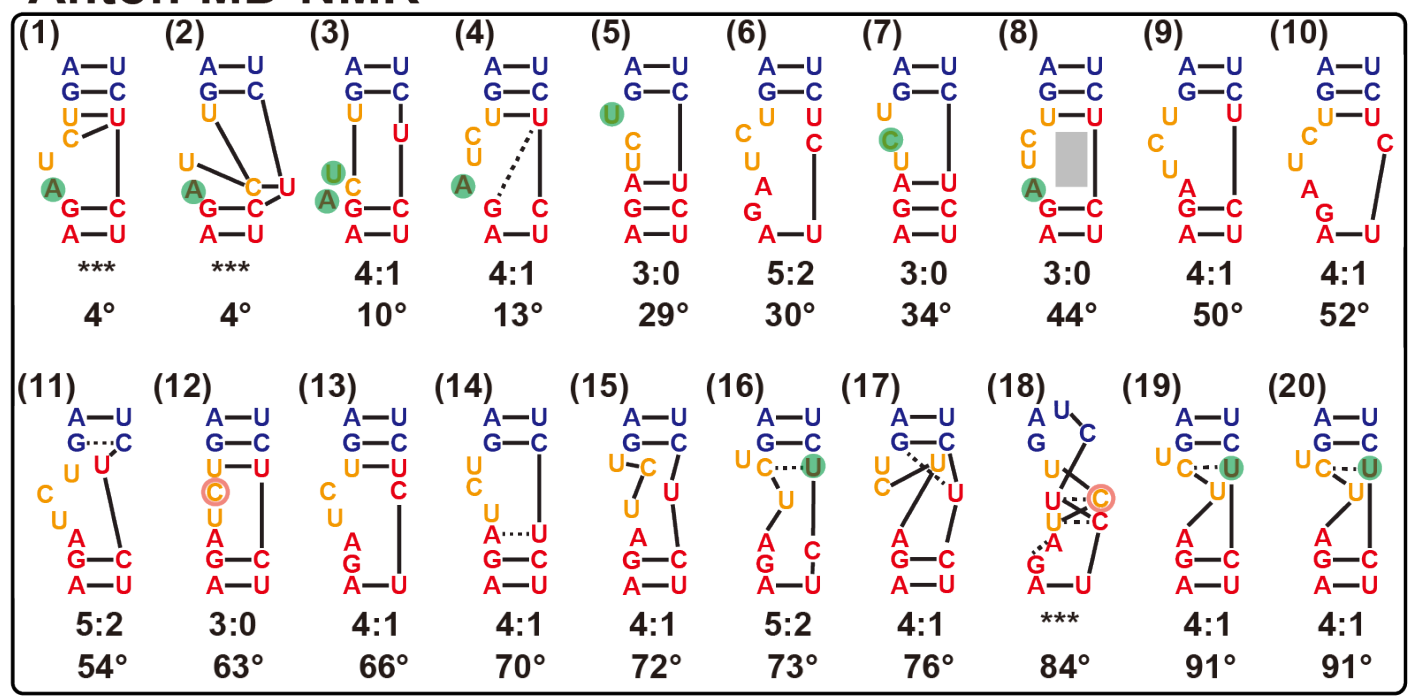

Extended Data Fig. 7 Junction topology scheme of the TAR ensembles. Junction topology in the (a) FARFAR-NMR $(N=20)$ and (b) Anton-MD-NMR $(N=20)$ ensembles. Conformers in each ensemble are sorted in increasing order of the bend angle magnitude $\left|\beta_{\mathrm{h}}\right|$, and the junction topology (Methods) as well as the $\left|\beta_{\mathrm{h}}\right|$ are labeled below each conformer. Junctional residues (bulge/A22-U40/G26-C39) with C2'-endo sugar pucker and non-gauche $+\gamma\left(\right.$ falling outside $\left.20-100^{\circ}\right)$ torsion angle are highlighted with green filled circle and orange open circle, respectively. 


\section{a U23}

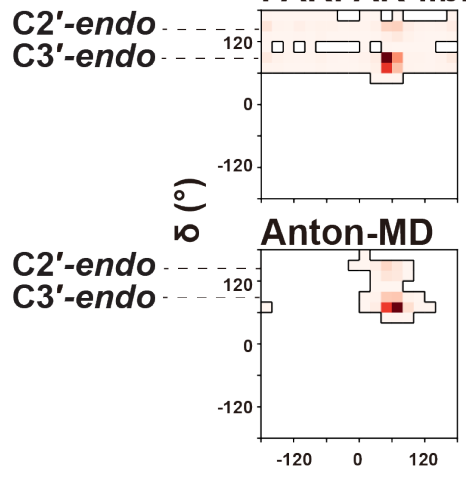

C24
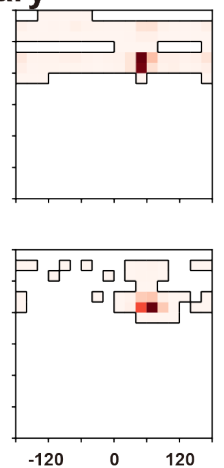

U25
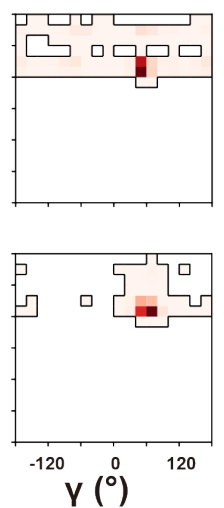
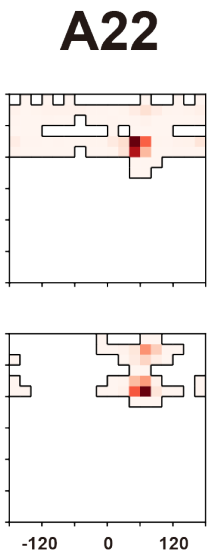

After SAS

b

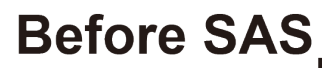

Random select
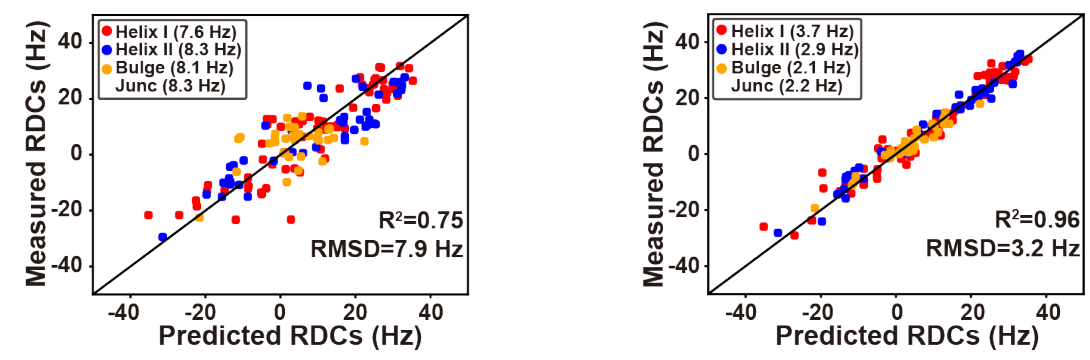

C

only C3'-endo
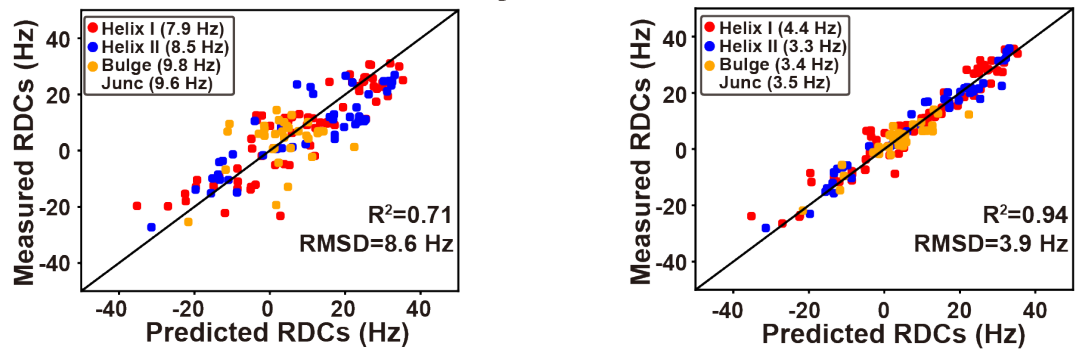

only gauche+
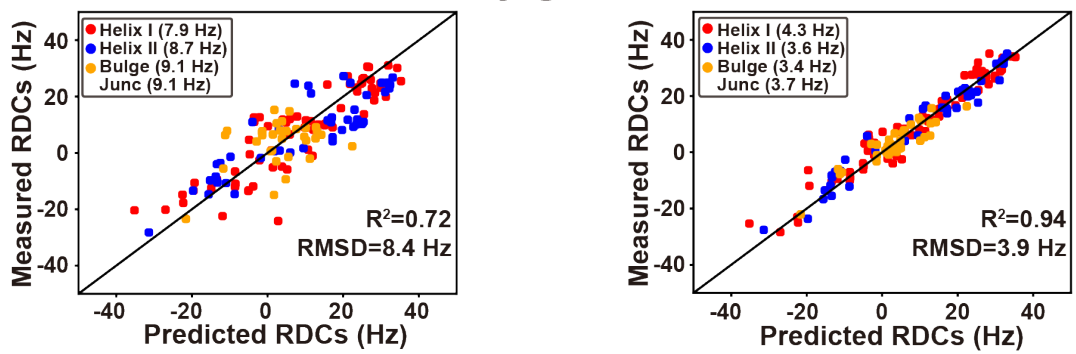

\section{Extended Data Fig. 8 Testing the sensitivity of FARFAR-library and FARFAR-NMR}

ensembles to variations in local torsion angles. (a) Distribution of backbone torsion angles of the FARFAR-library and Anton-MD. 2D density map of $\delta$ versus $\gamma$ of the bulge residues as well as A22 and U40, for the FARFAR-library and Anton-MD $(N=10,000)$. 
The bin width is $20^{\circ}$ for all density maps. (b-d) Comparison of measured and predicted RDCs for a subset of the FARFAR-library (left, $N=3,000$ ) and ensembles (right, $N=20$ ) following SAS on these subset libraries. (b) Randomly selected subset from the FARFAR-library (c) a subset of the FARFAR-library with the sugar puckers of U23, C24, U25, A22 and U40 chosen to be C3'-endo (d) a subset of the FARFAR-library with the $\gamma$ torsion angles of U23, C24, U25, A22 and U40 chosen to be gauche+ $\left(20^{\circ}<\gamma<100^{\circ}\right)$. "Junc" denotes bulge residues as well as A22 and U40. 


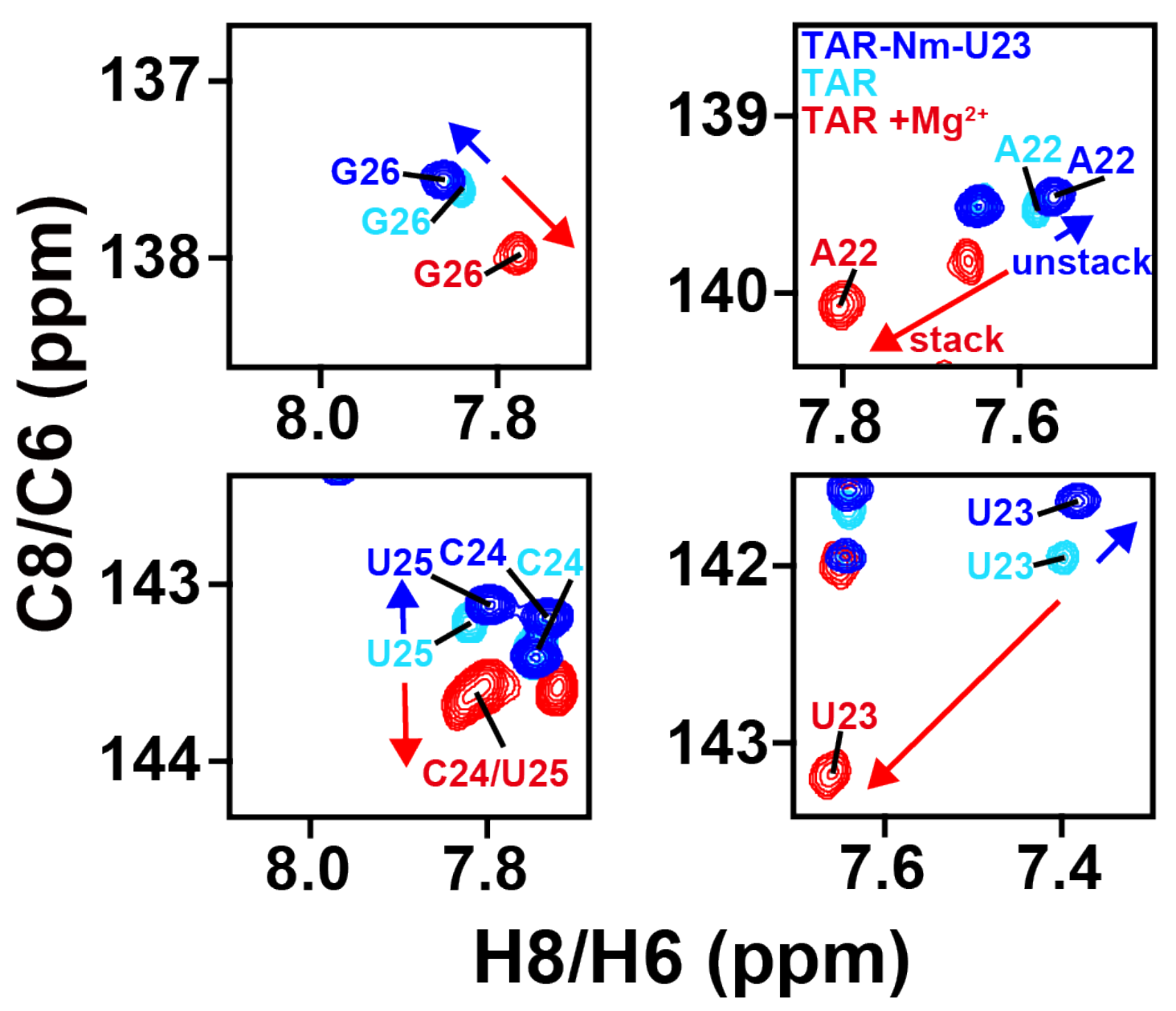

Extended Data Fig. 9 An additional Nm modified TAR showing Nm inducing unstack of TAR. Overlay of $2 \mathrm{D}\left[{ }^{13} \mathrm{C},{ }^{1} \mathrm{H}\right]$ HSQC NMR spectra of the aromatic region of TAR$\mathrm{Nm}-\mathrm{U} 23$ without $\mathrm{Mg}^{2+}$ (blue, inducing unstacking). TAR without $\mathrm{Mg}^{2+}$ (cyan), and TAR $+\mathrm{Mg}^{2+}$ (red, inducing stacking). 

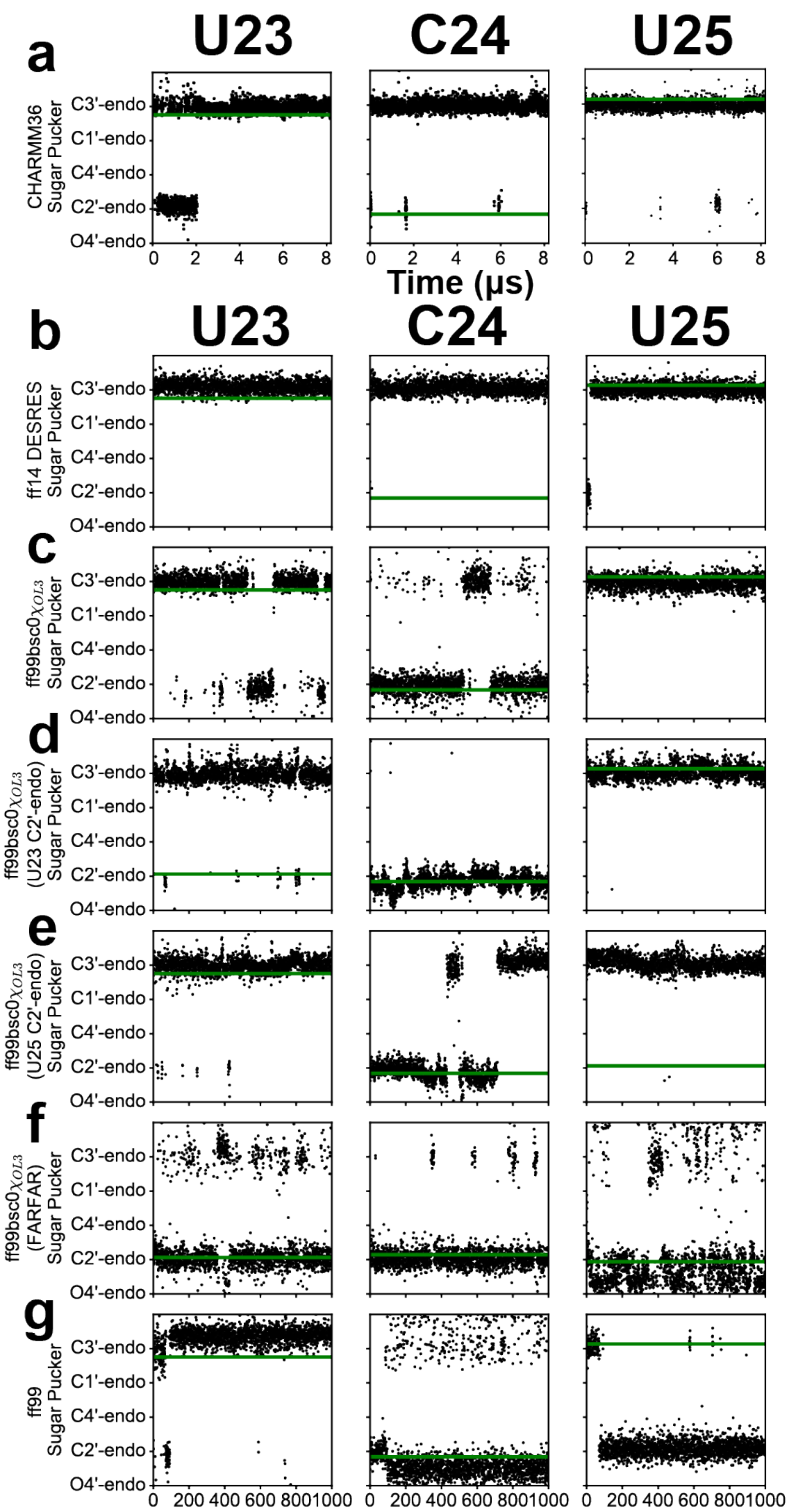

Time (ns) 
Extended Data Fig. 10 Sugar puckers of bulge nucleotides during the course of MD simulations with different force fields and starting structures. The variation of the sugar puckers of U23, C24 and U25 of TAR during the course of (a) an $8.2 \mu$ s trajectory using the Anton CHARMM36 force field and (b-g) a series of $1.0 \mu$ s trajectories using the (b) ff14 DESRES force field starting with the NOE structure (PDB: 1ANR), (c-f) ff99bsc0 $\chi$ OL3 force field starting with (c) the NOE structure (PDB 1ANR), (d) 1ANR with the pucker of $\mathrm{U} 23$ changed to $\mathrm{C}^{2}$-endo $\left(\mathrm{TAR}^{\mathrm{U} 23 \mathrm{C}^{\prime}-\text { endo }}\right)$, (e) $1 \mathrm{ANR}$ with the pucker of $\mathrm{U} 25$ sugar changed to $\mathrm{C} 2^{\prime}$-endo $\left(\mathrm{TAR}^{\mathrm{U} 25 \mathrm{C}^{\prime}-\text {-endo }}\right)$, (f) a FARFAR-NMR conformer in which all bulge nucleotides are $\mathrm{C} 2$ '-endo $\left(\mathrm{TAR}^{\mathrm{FARFAR}}\right)$, and $(\mathrm{g})$ the ff99 force field. The sugar pucker of the starting structures are indicated using a green line. Persistence of a general bias towards $\mathrm{C}^{\prime}$-endo or a tendency to maintain the sugar pucker in the initial starting conformation can be seen. 